\title{
An Age-Structured Model of HIV Latent Infection with Two Transmission Routes: Analysis and Optimal Control
}

\author{
Chunyang Qin, ${ }^{1}$ Xia Wang $\mathbb{D},{ }^{1,2}$ and Libin Rong ${ }^{3}$ \\ ${ }^{1}$ School of Mathematics and Statistics, Xinyang Normal University, Xinyang 464000, China \\ ${ }^{2}$ College of Mathematics and Information Science, Henan Normal University, Xinxiang 453000, China \\ ${ }^{3}$ Department of Mathematics, University of Florida, Gainesville, FL 32611, USA
}

Correspondence should be addressed to Xia Wang; xyxiawang@xynu.edu.cn

Received 4 March 2020; Accepted 13 July 2020; Published 24 August 2020

Academic Editor: Honglei Xu

Copyright ( $) 2020$ Chunyang Qin et al. This is an open access article distributed under the Creative Commons Attribution License, which permits unrestricted use, distribution, and reproduction in any medium, provided the original work is properly cited.

In addition to direct virus infection of target cells, HIV can also be transferred from infected to uninfected cells (cell-to-cell transmission). These two routes might facilitate viral production and the establishment of the latent virus pool, which is considered as a major obstacle to HIV cure. We studied an HIV infection model including the two infection routes and the time since latent infection. The basic reproductive ratio $R_{0}$ was derived. The existence, positivity, and boundedness of the solution are proved. We investigated the existence of steady states and their stability, which were shown to depend on $R_{0}$. We established the global asymptotic dynamical behavior by proving the existence of the global compact attractor and uniform persistence of the system and by applying the method of Lyapunov functionals. In the end, we formulated and solved the optimal control problem for the age-structured model. The necessary condition for minimization of the viral level and the cost of drug treatment was obtained, and numerical simulations of various optimal control strategies were performed.

\section{Introduction}

Human immunodeficiency virus (HIV) is a retrovirus that causes acquired immunodeficiency syndrome (AIDS) by infecting healthy $\mathrm{CD} 4^{+} \mathrm{T}$ cells, macrophages, and dendritic cells [1]. After infection, infected cells can generate new virus, which result in more infection and viral production. In order to investigate the within-host dynamics of HIV infection, many researchers have established different types of mathematical models (see, e.g., [2-11] and references cited therein). Most of these models focused on the infection of uninfected $\mathrm{CD}^{+}{ }^{+} \mathrm{T}$ cells by cell-free virus particles. In addition to cell-free viral infection, target cells can also be infected by virus transmitted directly from infected cells (cell-to-cell transmission) [12-20]. When infected $\mathrm{CD}^{+}$ $\mathrm{T}$ cells encounter uninfected $\mathrm{CD} 4^{+} \mathrm{T}$ cells and form viral synapses, HIV may spread by cell-to-cell transmission. The two routes of viral transmission may provide a synergistic way for virus infection $[2,21,22]$. The relative contributions of these two routes to viral production remain unclear. There is some evidence that HIV transmits more validly via cell-tocell transmission than cell-free virus infection [3, 23-30].

Models with age structure have been used in studying viral dynamics because many within-host processes, such as viral production by infected cells, may rely on the time after infection, i.e., the age of infection [31-41]. For example, Rong et al. [42] studied age of infection models with combination therapy including reverse transcriptase inhibitor (RTI), protease inhibitor (PI), and entry/fusion inhibitor. Wang et al. in [39] developed an HIV model with infection age and general nonlinear rates of viral infection in the infected $n$ types of target cells to study the transmission dynamics of HIV.

HIV latent infection of target cells remains to be a major barrier to viral clearance. To study the low viral persistence despite long-term antiretroviral therapy, mathematical models have been developed by incorporating cellular compartments or reservoirs such as latently infected $\mathrm{CD} 4^{+}$ T cells [43]. To explain the extremely slow decay of the latent viral pool and the viral persistence during therapy, 
Alshorman et al. [44] developed an HIV infection model involving latently infected cells with age structure. They showed that the model can generate a low viral load persistence and the extremely slow decay of the latent reservoir during prolonged therapy. In this work, we study an agestructured HIV model that includes two transmission routes to study virus dynamics. We will analyze the stability of the equilibria using the basic reproductive number. Because lifelong therapy is required for HIV-infected inhibitors, we formulate and solve the optimal control problem. The necessary condition for minimizing the viral level and the cost of drug treatment was obtained. Different optimal treatment strategies were evaluated by numerical investigations.

\section{The Model}

We introduce an HIV latent infection model with age structure and the two transmission routes. The model has four state variables: uninfected $\mathrm{CD} 4^{+}$target cells $T(t)$, agestructured latently infected cells, $L(t, a)$, infected cells that can produce virus, $I(t)$, and virus $V(t)$. The model can be expressed as follows:

$$
\left\{\begin{array}{l}
\frac{\mathrm{d} T(t)}{\mathrm{d} t}=\lambda-d_{T} T(t)-\beta T(t) V(t)-k T(t) I(t), \\
\frac{\partial L(t, a)}{\partial t}+\frac{\partial L(t, a)}{\partial a}=\rho(a) L(t, a)-\left(\alpha(a)+\delta_{L}(a)\right) L(t, a), \\
L(t, 0)=f \beta T(t) V(t)+f k T(t) I(t), \\
\frac{\mathrm{d} I(t)}{\mathrm{d} t}=(1-f)(\beta T(t) V(t)+k T(t) I(t))+\int_{0}^{\infty} \alpha(a) L(t, a) \mathrm{d} a-\delta I(t), \\
\frac{\mathrm{d} V(t)}{\mathrm{d} t}=N \delta I(t)-c V(t), \\
T(0)=T_{0} \in \mathbb{R}_{+}, \\
L(0, \cdot)=L_{0}(\cdot) \in L_{+}^{1}, \\
I(0)=I_{0} \in \mathbb{R}_{+}, \\
V(0)=V_{0} \in \mathbb{R}_{+} \cdot
\end{array}\right.
$$

The model assumes that $\lambda$ and $d_{T}$ are the recruitment rate and death rate of uninfected $\mathrm{CD} 4^{+} \mathrm{T}$ cells, respectively. The parameter $\beta$ is the infection rate of uninfected $\mathrm{CD} 4^{+}$ $\mathrm{T}$ cells by free virus and $k$ is the rate of transmission by infected cells. The constant $f \in(0,1)$ is the fraction of new infection that becomes latency. The rate of proliferation $\rho(a)$ and the rate of death $\delta_{L}(a)$ of latently infected cells are assumed to be functions of the age of infection. The activation rate $\alpha(a)$ also depends on the age because latently infected cells need to wait until they encounter their relevant antigen. The total number of productive infected cells $I(t)$ obtained by latently infected cell activation is given by $\int_{0}^{\infty} \alpha(a) L(t, a) \mathrm{d} a . \delta$ represents the death rate of infected cells. $N$ denotes the total number of virions released by one infected cell during its life cycle. The viral clearance rate is $c$. A schematic diagram of the full system is given in Figure 1.

To investigate the dynamics of (1), we need the following assumptions.
$\left(\mathrm{A}_{1}\right) L(0,0)=L_{0}(0)=f\left(\beta T_{0} V_{0}+k T_{0} I_{0}\right)$.

$\left(\mathrm{A}_{2}\right) \rho(\cdot), \alpha(\cdot), \delta_{L}(\cdot) \in L_{+}^{\infty}(0, \infty)$ are uniformly continuous, where $L_{+}^{\infty}(0, \infty)$ is the nonnegative cone of the Banach space $L^{\infty}(0, \infty)$.

The size of the latent reservoir remains relatively stable or declines extremely slowly during suppressive therapy [45]. Thus, the proliferation rate cannot exceed the sum of the activation rate and the death rate of latently infected cells. We have the following assumption as also used in [44].

$\left(\mathrm{A}_{3}\right)$ Because the latent reservoir is relatively stable during therapy, we let

$$
\sup _{a} \rho(a) \leq \inf _{a}\left(\alpha(a)+\delta_{L}(a)\right) .
$$

The state space of (1), 


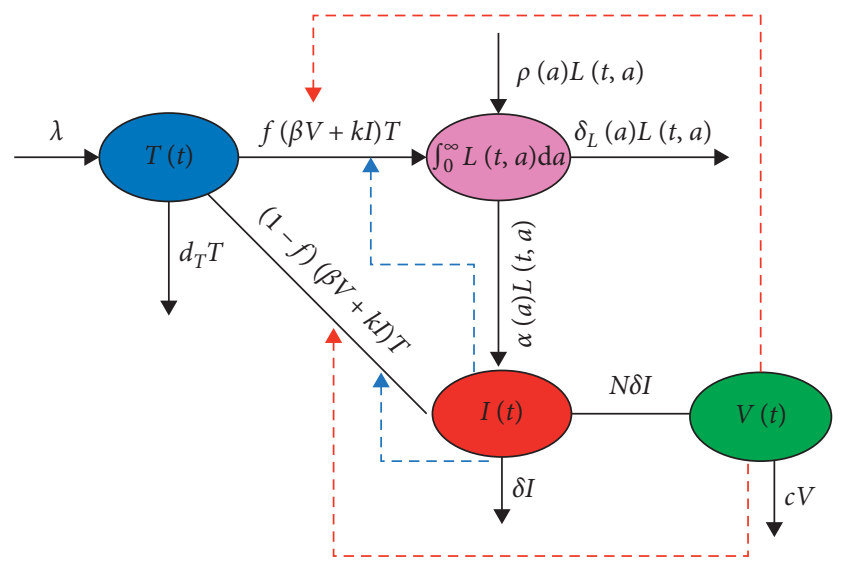

FIGURE 1: Schematic diagram of model (1).

$$
X_{+}=\mathbb{R}_{+} \times L_{+}^{1}(0, \infty) \times \mathbb{R}_{+} \times \mathbb{R}_{+},
$$

is the nonnegative cone of the Banach space $\mathbb{R} \times L^{1}(0, \infty) \times$ $\mathbb{R} \times \mathbb{R}$ equipped with the norm

$$
\|(T, L, I, V)\|=|T|+\|L\|_{1}+|I|+|V|,
$$

for $(T, L, I, V) \in \mathbb{R} \times L^{1}(0, \infty) \times \mathbb{R} \times \mathbb{R}$.

From Iannelli [46] and Magal [47], if the initial condition $\left(T_{0}, L_{0}, I_{0}, V_{0}\right) \in X_{+}$satisfies $\left(\mathrm{A}_{1}\right)$, then system (1) has a unique continuous solution in $X_{+}$. Therefore, we can define a solution semiflow $\Phi: \mathbb{R}_{+} \times X_{+} \longrightarrow X_{+}$of (1) by $\Phi\left(t,\left(T_{0}, L_{0}, I_{0}, V_{0}\right)\right)=(T(t), L(t, \cdot), I(t), V(t))$.

Let

$$
\begin{aligned}
& F_{1}(t)=T(t)+\int_{0}^{\infty} L(t, a) \mathrm{d} a, \\
& F_{2}(t)=T(t)+I(t) .
\end{aligned}
$$

It follows from (1) that

$$
\begin{aligned}
\frac{\mathrm{d} F_{1}(t)}{\mathrm{d} t} & \leq \lambda-\mathrm{d}_{T} T-\int_{0}^{\infty}\left(\alpha(a)+\delta_{L}(a)-\rho(a)\right) L(t, a) \mathrm{d} a \\
& =\lambda-d_{T} T-\int_{0}^{\infty} m(a) L(t, a) \mathrm{d} a \\
& \leq \lambda-\mu_{1} F_{1}(t),
\end{aligned}
$$

where $\quad m(a)=\alpha(a)+\delta_{L}(a)-\rho(a), \quad \mu_{1}=\min \left\{d_{T}, m_{0}\right\}$, and $m_{0}=\inf _{a \in(0, \infty)}\{m(a)\}$. This leads to $\lim \sup _{t \longrightarrow \infty} F_{1}$ $(t) \leq \lambda / \mu_{1}$.

$$
\begin{aligned}
\frac{\mathrm{d} F_{2}(t)}{\mathrm{d} t} & \leq \lambda-\mathrm{d}_{T} T+\int_{0}^{\infty} \alpha(a) L(t, a) \mathrm{d} a-\delta I \\
& \leq \lambda+\|\alpha\|^{\infty} \frac{\lambda}{\mu_{1}}-\mathrm{d}_{T} T-\delta I \\
& \leq \lambda+\|\alpha\|^{\infty} \frac{\lambda}{\mu_{1}}-\mu_{2} F_{2}(t),
\end{aligned}
$$

which means that limsup $t \rightarrow \infty F_{2}(t) \leq \lambda / \mu_{2}+\|\alpha\|^{\infty} \lambda /\left(\mu_{1} \mu_{2}\right)$, where $\mu_{2}=\min \left\{d_{T}, \delta\right\}$.

Because

$$
\begin{aligned}
\frac{\mathrm{d} V(t)}{\mathrm{d} t} & =N \delta I-c V \\
& \leq N \delta\left(\frac{\lambda}{\mu_{2}}+\|\alpha\|^{\infty} \frac{\lambda}{\mu_{1} \mu_{2}}\right)-c V,
\end{aligned}
$$

we obtain $\limsup _{t \rightarrow \infty} V(t) \leq(N \delta / c)\left(\left(\lambda / \mu_{2}\right)+\|\alpha\|^{\infty} \lambda /\right.$ $\left.\left(\mu_{1} \mu_{2}\right)\right)$.

The set

$$
\Omega=\left\{(T, L, I, V) \in X_{+} \mid T+\|L\|_{1} \leq \frac{\lambda}{\mu_{1}}, T+I \leq \frac{\lambda}{\mu_{2}}+\|\alpha\|^{\infty} \frac{\lambda}{\mu_{1} \mu_{2}}, V \leq \frac{N \delta}{c}\left(\frac{\lambda}{\mu_{2}}+\|\alpha\|^{\infty} \frac{\lambda}{\mu_{1} \mu_{2}}\right)\right\}
$$

is a positive invariant and attracting subset for system (1).

\section{Analysis of Model (1)}

3.1. The Existence of Equilibria and Basic Reproduction Number. System (1) always has an infection-free equilibrium $E^{0}=\left(T^{0}, 0,0,0\right)$, where $T^{0}=\lambda / d_{T}$.

Denote

$$
\begin{aligned}
\xi & =\int_{0}^{\infty} \alpha(a) \sigma(a) \mathrm{d} a \\
\sigma(a) & =\exp \left(-\int_{0}^{a} m(s) \mathrm{d} s\right), \quad \text { for } a \in \mathbb{R}_{+},
\end{aligned}
$$

where $m(s)=\alpha(s)+\delta_{L}(s)-\rho(s)$.

Define

$$
\begin{aligned}
R_{0} & =\left(\frac{N \beta \lambda}{c d_{T}}+\frac{k \lambda}{\delta d_{T}}\right)(f \xi+1-f) \\
& =\frac{N \beta \lambda}{c d_{T}}(f \xi+1-f)+\frac{k \lambda}{\delta d_{T}}(f \xi+1-f) \\
& \triangleq R_{01}+R_{02}
\end{aligned}
$$

where $R_{0}$ is the basic reproductive ratio of model (1) and $R_{01}$ is the portion from cell-free virus infection while $R_{02}$ is from the other transmission route (see details in [40]).

When $R_{0}$ is greater than 1 , the model has a positive equilibrium, given by $E^{*}=\left(T^{*}, L^{*}, I^{*}, V^{*}\right) \in \Omega$, which satisfies 


$$
\left\{\begin{array}{l}
\lambda-d_{T} T^{*}-\beta T^{*} V^{*}-k T^{*} I^{*}=0, \\
\frac{\mathrm{d}}{\mathrm{d} a} L^{*}(a)=-m(a) L^{*}(a), \\
L^{*}(0)=f \beta T^{*} V^{*}+f k T^{*} I^{*}, \\
(1-f) \beta T^{*} V^{*}+(1-f) k T^{*} I^{*}+\xi L^{*}(0)-\delta I^{*}=0, \\
N \delta I^{*}=c V^{*} .
\end{array}\right.
$$

Straightforward calculation yields the infected equilibrium:

$$
\Delta(\bar{E})=\mid \begin{array}{cc}
\tau+d_{T}+\beta \bar{V}+k \bar{I} & 0 \\
f \beta \bar{V}+f k \bar{I} & -1 \\
-(1-f)(\beta \bar{V}+k \bar{I}) & -\xi(\tau) \\
0 & 0
\end{array}
$$

where $\tau$ denotes the eigenvalue of the characteristic equation (14) and $\xi(\tau)=\int_{0}^{\infty} \alpha(a) \sigma(a) e^{-\tau a} \mathrm{~d} a$. Obviously, the equilibrium $\bar{E}$ of (1) is LAS (i.e., locally asymptotically stable) if the real parts of the roots of equation (14) are all negative; if at least one eigenvalue of (14) has a positive real part, then the equilibrium $\bar{E}$ of (1) is unstable.

Theorem 1. The infection-free equilibrium $E^{0}$ of system (1) is LAS when $R_{0}<1$ and it is unstable when $R_{0}>1$.

Proof. The characteristic equation of equilibrium $E^{0}$ of system (1) can be simplified as

$$
\Delta\left(E^{0}\right)=\left(\tau+d_{T}\right)(\tau+\delta)(\tau+c) h_{1}(\tau)=0,
$$

$$
\left\{\begin{array}{l}
T^{*}=\frac{c \delta}{N \delta \beta(f \xi+(1-f))+k c(f \xi+(1-f))}=\frac{\lambda}{d_{T} R_{0}} \\
L^{*}(a)=\frac{\lambda\left(R_{0}-1\right) f}{R_{0}} \sigma(a), \\
I^{*}=\frac{c d_{T}\left(R_{0}-1\right)}{N \delta \beta+k c} \\
V^{*}=\frac{N \delta d_{T}\left(R_{0}-1\right)}{N \delta \beta+k c} .
\end{array}\right.
$$

3.2. Local Stability. Let $\bar{E}=(\bar{T}, \bar{L}, \bar{I}, \bar{V})$ be any equilibrium of (1). After linearizing (1) at $\bar{E}$, we get the characteristic equation as follows:

where

$$
\begin{aligned}
h_{1}(\tau)= & 1-\frac{k T^{0}\left(1-f+f \int_{0}^{\infty} \alpha(a) \sigma(a) e^{-\tau a} \mathrm{~d} a\right)}{\tau+\delta} \\
& -\frac{N \delta \beta T^{0}\left(1-f+f \int_{0}^{\infty} \alpha(a) \sigma(a) e^{-\tau a} \mathrm{~d} a\right)}{(\tau+\delta)(\tau+c)} .
\end{aligned}
$$

Therefore, the stability of $E^{0}$ is decided by the roots of the equation $h_{1}(\tau)=0$. When $R_{0}<1$, we want to show that the real parts of all the roots of $h_{1}(\tau)=0$ are negative. It is proved by contradiction. Let $\tau^{0}$ be a root of $h_{1}(\tau)=0$ with nonnegative real part. It follows from equation (16) that

$$
\begin{aligned}
1 & =\left|\frac{k T^{0}\left(1-f+f \int_{0}^{\infty} \alpha(a) \sigma(a) e^{-a \tau^{0}} \mathrm{~d} a\right)}{\tau^{0}+\delta}+\frac{N \delta \beta T^{0}\left(1-f+f \int_{0}^{\infty} \alpha(a) \sigma(a) e^{-a \tau^{0}} \mathrm{~d} a\right)}{\left(\tau^{0}+\delta\right)\left(\tau^{0}+c\right)}\right| \\
& \leq \frac{k T^{0}(1-f+f \xi)}{\delta}+\frac{N \delta \beta T^{0}(1-f+f \xi)}{\delta c} \\
& =R_{0},
\end{aligned}
$$

which leads to a contradiction since $R_{0}<1$. Thus, we know that each root of equation (15) is negative or has negative real part. Therefore, infection-free equilibrium $E^{0}$ is LAS when $R_{0}<1$. 
If $R_{0}>1$, we have $h_{1}(0)=1-R_{0}<0$. From $\lim _{\tau \longrightarrow \infty} h_{1}(\tau)=1>0$, the equation $h_{1}(\tau)=0$ has at least one positive real root, which means that the equilibrium $E^{0}$ is unstable for $R_{0}>1$.

Next, we show that when $R_{0}>1$, the positive equilibrium $E^{*}$ is LAS.

Theorem 2. When $R_{0}>1$, the infected equilibrium $E^{*}$ of (1) is LAS.

Proof. Using the equilibrium equality $d_{T} R_{0}=d_{T}+\beta V^{*}+$ $k I^{*}$ and equation (14), we have the characteristic equation at $E^{*}$ :

$$
\begin{aligned}
\Delta\left(E^{*}\right)= & \left(\tau+d_{T} R_{0}\right)(\tau+c)(\tau+\delta)-\left(\tau+d_{T}\right) \\
& \cdot\left(k T^{*}(\tau+c)+N \delta \beta T^{*}\right) \\
& \cdot\left(1-f+f \int_{0}^{\infty} \alpha(a) \sigma(a) e^{-\tau a} \mathrm{~d} a\right)=0 .
\end{aligned}
$$

We now claim that equation (18) has no root with a nonnegative real part. If it is not, let us assume that it has a root $\tau_{0}$ with $\operatorname{Re}\left(\tau_{0}\right) \geq 0$. Equation (18) becomes the following by dividing $\left(\tau_{0}+d_{T} R_{0}\right)\left(\tau_{0}+c\right)\left(\tau_{0}+\delta\right)$ :

$$
1=\frac{\left(\tau_{0}+d_{T}\right)\left(k T^{*}\left(\tau_{0}+c\right)+N \delta \beta T^{*}\right)\left(1-f+f \int_{0}^{\infty} \alpha(a) \sigma(a) e^{-\tau_{0} a} \mathrm{~d} a\right)}{\left(\tau_{0}+d_{T} R_{0}\right)\left(\tau_{0}+c\right)\left(\tau_{0}+\delta\right)}
$$

Taking the modulus of the two sides of equation (19), we have

$$
\begin{aligned}
1 & <\frac{k T^{*}(1-f+f \xi)}{\delta}+\frac{N \beta T^{*}(1-f+f \xi)}{c} \\
& =\frac{R_{0}}{T^{0}} \cdot T^{*}=1,
\end{aligned}
$$

which is a contradiction. Therefore, the equilibrium $E^{*}$ is LAS when $R_{0}>1$.

\section{Global Dynamics of (1)}

In the present section, by using suitable Lyapunov functionals, we establish the global asymptotic dynamics of the equilibria of (1). The results acquired in the following indicate that the GAS (i.e., global asymptotic stability) is completely determined by the basic reproduction number. We start with the global stability of $E^{0}$ by applying the method of Lyapunov functionals when $R_{0}<1$ (see proof in Appendix A).

Theorem 3. The infection-free equilibrium $E^{0}$ of (1) is GAS when $R_{0}<1$.

To prove the equilibrium $E^{*}$ of (1) is GAS, we need some preparation.

Let $\rho: \Omega \longrightarrow \mathbb{R}_{+}$by

$$
\rho(T, L, I, V)=\beta T(t) V(t)+k T(t) I(t)+\int_{0}^{\infty} \alpha(a) L(t, a) \mathrm{d} a,
$$

for $(T, L, I, V) \in \Omega$. Define

$\Omega_{0}=\left\{\left(T_{0}, L_{0}, I_{0}, V_{0}\right) \in \Omega \mid\right.$ There is $t_{0} \in \mathbb{R}_{+}$ensuring $\left.\rho\left(\Phi\left(t_{0}, x_{0}\right)\right)>0\right\}$.

Given any $x_{0}=\left(T_{0}, L_{0}, I_{0}, V_{0}\right) \in \Omega \backslash \Omega_{0}$, we have $\lim _{t \rightarrow \infty} \Phi\left(t, x_{0}\right)=E^{0}$. From Lemma 3.2 of Hale [48] and
Theorem 3.2 of Thieme [49], one can obtain the following results with standard arguments (also see Chen et al. [50]).

Theorem 4. Suppose $R_{0}>1$. Then, the following statements are true. (i) There exists a global attractor $\mathscr{A}$ for the solution semiflow $\Phi$ of (1) in $\Omega_{0}$. (ii) System (1) is uniformly strongly $\rho$-persistent, that is, there exists $\varepsilon_{0}>0$ (independent of initial values) such that

$$
\liminf _{t \longrightarrow \infty} \rho(\Phi(t, x))>\varepsilon_{0}, \quad \text { for } x \in \Omega_{0} .
$$

It can only contain points that pass through their total trajectories because the global attractor $\mathscr{A}$ is invariant. A total trajectory of $\Phi$ is a function $\omega: \mathbb{R} \longrightarrow X_{+}$ensuring that $\Phi(s, \omega(t))=\omega(s+t)$ for all $t \in \mathbb{R}$ and $s \in \mathbb{R}_{+}$. For a total trajectory, $L(t, a)=L(t-a, 0) \sigma(a)$ for all $a \in \mathbb{R}_{+}$and $t \in \mathbb{R}$. The alpha limit of a total trajectory $\omega(t)$ passing through $\omega(0)=\omega_{0}$ is

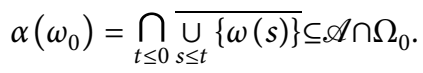

Corollary 1. We assume that $R_{0}>1$. Then, there exists $\varepsilon_{0}>0$ such that $T, L, I, V \geq \varepsilon_{0}$ for all $t \in \mathbb{R}$, where $(T(t), L(t, 0), I(t), V(t))$ is any total trajectory in $\mathscr{A}$.

Proof. Since $\Omega$ is attracting and invariant, there is $t_{1} \in \mathbb{R}_{+}$ ensuring that

$$
\begin{aligned}
I(t) & \leq \frac{\lambda+\|\alpha\|^{\infty}\left(\lambda / \mu_{1}\right)}{\mu_{2}}, \\
V(t) & \leq \frac{N \delta\left(\lambda+\|\alpha\|^{\infty}\left(\lambda / \mu_{1}\right)\right)}{c \mu_{2}}, \text { for } t \geq t_{1} .
\end{aligned}
$$

For $t \geq t_{1}$, from the first equation of system (1), we get $\frac{\mathrm{d} T(t)}{\mathrm{d} t} \geq \lambda-\left(d_{T}+\beta \frac{N \delta\left(\lambda+\|\alpha\|^{\infty}\left(\lambda / \mu_{1}\right)\right)}{c \mu_{2}}+k \frac{\lambda+\|\alpha\|^{\infty}\left(\lambda / \mu_{1}\right)}{\mu_{2}}\right) T(t)$, 
which leads to

$$
\liminf _{t \rightarrow \infty} T(t) \geq \frac{\lambda}{d_{T}+\beta N \delta\left(\lambda+\|\alpha\|^{\infty}\left(\lambda / \mu_{1}\right)\right) /\left(c \mu_{2}\right)+k\left(\lambda+\|\alpha\|^{\infty}\left(\lambda / \mu_{1}\right)\right) / \mu_{2}} \stackrel{\Delta}{=} \varepsilon_{T} .
$$

By invariance, $T(t) \geq \varepsilon_{T}$ for all $t \in \mathbb{R}$.

It follows from Theorem 4 that there is $\bar{\varepsilon}>0$ ensuring that

$\int_{0}^{\infty} \alpha(a) L(t, a) \mathrm{d} a+\beta T(t) V(t)+k T(t) I(t) \geq \bar{\varepsilon}, \quad$ for $t \in \mathbb{R}$.

This, combined with system (1), gives

$$
\frac{\mathrm{d} I(t)}{\mathrm{d} t} \geq \bar{\varepsilon}-\delta I(t), \quad \text { for } t \in \mathbb{R} .
$$

Therefore, we have

$$
\liminf _{t \longrightarrow \infty} I(t) \geq \frac{\bar{\varepsilon}}{\delta} \triangleq \varepsilon_{I}
$$

and thus $I(t) \geq \varepsilon_{I}$ for $t \in \mathbb{R}$ by invariance again.

It follows from the last equation of (1) that

$$
\liminf _{t \rightarrow \infty} V(t) \geq \frac{N \delta \varepsilon_{I}}{c} \triangleq \varepsilon_{V} .
$$

Thus,

$L(t, 0)=f \beta T V+f k T I \geq f \beta \varepsilon_{T} \varepsilon_{v}+f \varepsilon_{T} \varepsilon_{I} \triangleq \varepsilon_{L}, \quad$ for $t \in \mathbb{R}$.

Letting $\varepsilon_{0}=\min \left\{\varepsilon_{T}, \varepsilon_{L}, \varepsilon_{I}, \varepsilon_{V}\right\}$, we complete the proof.

The following theorem establishes the GAS of $E^{*}$ using the methods of Lyapunov functionals when $R_{0}>1$. The proof is given in Appendix $\mathrm{B}$.

Theorem 5. The infected equilibrium $E^{*}$ of system (1) is GAS when $R_{0}>1$.

\section{The Optimal Control Problem}

To obtain the optimal treatment strategies, we study the following model with controls

$$
\left\{\begin{array}{l}
\frac{\mathrm{d} T(t)}{\mathrm{d} t}=\lambda-d_{T} T(t)-\left(1-\varepsilon_{1}(t)\right) \beta T(t) V(t)-\left(1-\varepsilon_{1}(t)\right) k T(t) I(t), \\
\frac{\partial L(t, a)}{\partial t}+\frac{\partial L(t, a)}{\partial a}=\rho(a) L(t, a)-\left(\alpha(a)+\delta_{L}(a)\right) L(t, a) \\
L(t, 0)=\left(1-\varepsilon_{1}(t)\right) f \beta T(t) V(t)+\left(1-\varepsilon_{1}(t)\right) f k T(t) I(t), \\
\frac{\mathrm{d} I(t)}{\mathrm{d} t}=(1-f)\left(1-\varepsilon_{1}(t)\right)(\beta T(t) V(t)+k T(t) I(t))+\int_{0}^{\infty} \alpha(a) L(t, a) \mathrm{d} a-\delta I(t) \\
\frac{\mathrm{d} V(t)}{\mathrm{d} t}=\left(1-\varepsilon_{2}(t)\right) N \delta I(t)-c V(t), \\
T(0)=T_{0} \in \mathbb{R}_{+} \\
L(0, \cdot)=L_{0}(\cdot) \in L_{+}^{1} \\
I(0)=I_{0} \in \mathbb{R}_{+}, \\
V(0)=V_{0} \in \mathbb{R}_{+} \cdot
\end{array}\right.
$$

One control term $\varepsilon_{1}(t)$ is the effectiveness of RTIs, which block new infection. The other control term $\varepsilon_{2}(t)$ denotes the effectiveness of PIs, which reduce the number of infectious virions. 
We minimize the objective functional

$$
J\left(\varepsilon_{1}, \varepsilon_{2}\right)=\int_{0}^{t_{f}}\left(r_{1} \varepsilon_{1}^{2}(t)+r_{2} \varepsilon_{2}^{2}(t)+q V(t)\right) \mathrm{d} t,
$$

where $t_{f}$ is the duration of the treatment and $r_{1}, r_{2}, q$ are the positive weight coefficients to balance the control functions and the quantity of virus particles. The aim of this section is to minimize the objective functional defined in equation (34) by decreasing the viral load and the cost of drug treatment. The two control functions $\varepsilon_{1}(t)$ and $\varepsilon_{2}(t)$ represent the efficacy of RTIs and PIs satisfying $0 \leq \varepsilon_{i, \min } \leq \varepsilon_{i}(t) \leq \varepsilon_{i, \max }<1, i=1,2$.

Problem 1. We seek optimal controls $\varepsilon_{1}^{*}$ and $\varepsilon_{2}^{*}$ such that

$$
J\left(\varepsilon_{1}^{*}, \varepsilon_{2}^{*}\right)=\min \left\{J\left(\varepsilon_{1}, \varepsilon_{2}\right) \mid \varepsilon_{1}(t) \text { and } \varepsilon_{2}(t) \text { are Lebesque-integrable on }\left[0, t_{f}\right] \text { with values in } U_{i}=\left[\varepsilon_{i, \min }, \varepsilon_{i, \max }\right], i=1,2\right\}
$$

subject to system (33) with the boundary condition

$$
L(t, 0)=f\left(1-\varepsilon_{1}(t)\right) \beta T(t) V(t)+f\left(1-\varepsilon_{1}(t)\right) k T(t) I(t),
$$

and the initial values

$$
\begin{aligned}
T(0) & =T_{0}, \\
L(0, a) & =L_{0}(a), \\
I(0) & =I_{0}, \\
V(0) & =V_{0} .
\end{aligned}
$$

We noticed that the existence of optimal control functions to Problem 1 can be obtained by the methods used in $[51,52]$.

By the discretization-differential approach [52], we divide $L(t, a)$ into a number of classes from age 0 to $a_{\max }$, changing the age-structure model (33) to ODEs. We differentiate the ODEs to get an adjoint (costate) system. Let $\left\{a_{j}\right\}_{j=0}^{n}$ be a partition of $\left[0, a_{\max }\right]$ with $a_{0}=0, a_{j-1} \leq a_{j}, \Delta a_{j}=a_{j}-a_{j-1}(j=1,2, \ldots, n), \quad$ and $a_{n}=a_{\max }$. The discrete system is

$$
\frac{\mathrm{d} T(t)}{\mathrm{d} t}=\lambda-d_{T} T(t)-\left(1-\varepsilon_{1}(t)\right) \beta T(t) V(t)
$$

$$
-\left(1-\varepsilon_{1}(t)\right) k T(t) I(t)
$$

$$
\frac{\mathrm{d} L}{\mathrm{~d} t}\left(t, a_{j}\right)=-\frac{L\left(t, a_{j}\right)-L\left(t, a_{j-1}\right)}{\Delta a_{j}}-m\left(a_{j}\right) L\left(t, a_{j}\right),
$$

$$
\begin{aligned}
\frac{\mathrm{d} I(t)}{\mathrm{d} t}= & (1-f)\left(1-\varepsilon_{1}(t)\right)(\beta T(t) V(t)+k T(t) I(t)) \\
& +\sum_{j=1}^{n} \alpha\left(a_{j}\right) L\left(t, a_{j}\right) \Delta a_{j}-\delta I(t)
\end{aligned}
$$$$
\frac{\mathrm{d} V(t)}{\mathrm{d} t}=\left(1-\varepsilon_{2}(t)\right) N \delta I(t)-c V(t),
$$$$
L(t, 0)=\left(1-\varepsilon_{1}(t)\right) f(\beta T(t) V(t)+k T(t) I(t)),
$$

$$
L\left(0, a_{j}\right)=L_{0}\left(a_{j}\right) .
$$

Let $L_{j}=L\left(t, a_{j}\right)$ and $X=\left(T, L_{1}, L_{2}, \ldots, L_{n}, I, V\right)^{\mathrm{T}}$. The constraint equations are given by

$$
\dot{X}=\left(\begin{array}{c}
T \\
L_{1} \\
L_{2} \\
\cdots \\
L_{n} \\
I \\
V
\end{array}\right)^{\prime}=\left(\begin{array}{c}
\lambda-d_{T} T-\left(1-\varepsilon_{1}\right)(\beta T V+k T I) \\
-\frac{L_{1}-\left(1-\varepsilon_{1}\right) f(\beta T V+k T I)}{\Delta a_{1}}-m\left(a_{1}\right) L_{1} \\
-\frac{L_{2}-L_{1}}{\Delta a_{2}}-m\left(a_{2}\right) L_{2} \\
\ldots \\
(1-f)\left(1-\varepsilon_{1}\right)(\beta T V+k T I)+\sum_{j=1}^{n} \alpha\left(a_{j}\right) L_{j} \Delta a_{j}-\delta I \\
\Delta a_{n-1}-m\left(a_{n}\right) L_{n} \\
\left(1-\varepsilon_{2}\right) N \delta I-c V
\end{array}\right) .
$$


In the following, using the Pontryagin maximum principle [53], we will obtain the optimal control functions $\varepsilon_{1}^{*}$ and $\varepsilon_{2}^{*}$. To achieve this, we introduce a Lagrange multiplier or adjoint variable $Y=\left(\omega(t), \lambda_{1}(t), \lambda_{2}(t), \ldots, \lambda_{n}(t), \omega_{1}(t), \omega_{2}(t)\right)^{\mathrm{T}}$ and define the Lagrangian $\mathscr{L}$ as follows:

$$
\begin{aligned}
\mathscr{L}= & r_{1} \varepsilon_{1}^{2}+r_{2} \varepsilon_{2}^{2}+q V \\
& +\omega\left[\lambda-d_{T} T-\left(1-\varepsilon_{1}(t)\right)(\beta T V+k T I)\right] \\
& +\lambda_{1}\left(-\frac{L_{1}}{\Delta a_{1}}+\frac{\left(1-\varepsilon_{1}\right) f(\beta T V+k T I)}{\Delta a_{1}}-m\left(a_{1}\right) L_{1}\right) \\
& +\sum_{j=2}^{n} \lambda_{j}\left(-\frac{L_{j}-L_{j-1}}{\Delta a_{j}}-m\left(a_{j}\right) L_{j}\right) \\
& +\omega_{1}\left((1-f)\left(1-\varepsilon_{1}\right)(\beta T V+k T I)+\sum_{j=1}^{n} \alpha\left(a_{j}\right) L_{j} \Delta a_{j}-\delta I\right) \\
& +\omega_{2}\left(\left(1-\varepsilon_{2}\right) N \delta I-c V\right) \\
& -\xi_{1}\left(\varepsilon_{1}-\varepsilon_{1, \min }\right)-\eta_{1}\left(\varepsilon_{1, \max }-\varepsilon_{1}\right) \\
& -\xi_{2}\left(\varepsilon_{2}-\varepsilon_{2, \min }\right)-\eta_{2}\left(\varepsilon_{2, \max }-\varepsilon_{2}\right),
\end{aligned}
$$

where $\xi_{i}(t) \geq 0, \eta_{i}(t) \geq 0(i=1,2)$ are the penalty multipliers satisfying $\xi_{i}(t)\left(\varepsilon_{i}-\varepsilon_{i, \min }\right)=\eta_{i}(t)\left(\varepsilon_{i, \max }-\varepsilon_{i}\right)(i=1,2)$ at $\varepsilon_{i}=\varepsilon_{i}^{*}$ and $\varepsilon_{1}^{*}, \varepsilon_{2}^{*}$ are the optimal controls.

Theorem 6. Given optimal controls and solutions with respect to the corresponding constraint equation (1) that minimize the objective functional (34), there exists an adjoint variable $Y=\left(\omega(t), \lambda_{1}(t), \lambda_{2}(t), \ldots, \lambda_{n}(t), \omega_{1}(t), \omega_{2}(t)\right)^{T}$ satisfying

$$
\dot{Y}=\left(\begin{array}{c}
\omega \\
\lambda_{1} \\
\lambda_{2} \\
\cdots \\
\lambda_{n} \\
\omega_{1} \\
\omega_{2}
\end{array}\right)^{\prime}=-\left(\begin{array}{c}
-d_{T} \omega-\left(1-\varepsilon_{1}\right)(\beta V+k I)\left(\omega-\frac{f \lambda_{1}}{\Delta a_{1}}-(1-f) \omega_{1}\right) \\
-\frac{\lambda_{1}}{\Delta a_{1}}-m\left(a_{1}\right) \lambda_{1}+\frac{\lambda_{2}}{\Delta a_{2}}+\alpha\left(a_{1}\right) \Delta a_{1} \omega_{1} \\
-\frac{\lambda_{2}}{\Delta a_{2}}-m\left(a_{2}\right) \lambda_{2}+\frac{\lambda_{3}}{\Delta a_{3}}+\alpha\left(a_{2}\right) \Delta a_{2} \omega_{1} \\
\cdots \\
-\left(1-\varepsilon_{1}\right) k T\left(\omega-\frac{f \lambda_{1}}{\Delta a_{1}}-(1-f) \omega_{1}\right)-\delta \omega_{1}+\left(1-\varepsilon_{2}\right) N \delta \omega_{2} \\
q-\left(1-\varepsilon_{1}\right) \beta T\left(\omega-\frac{f \lambda_{1}}{\Delta a_{1}}-(1-f) \omega_{1}\right)-c \omega_{2}
\end{array}\right),
$$

with the terminal conditions $Y\left(t_{f}\right)=(0,0,0, \ldots, 0,0,0)^{T}$. Furthermore, the optimal control functions $\varepsilon_{1}^{*}$ and $\varepsilon_{2}^{*}$ are given by

$$
\begin{aligned}
& \varepsilon_{1}^{*}=\max \left\{\varepsilon_{1, \min }, \min \left\{\varepsilon_{1, \max }, \frac{-\omega(t)+\left(f / \Delta a_{1}\right) \lambda_{1}(t)+(1-f) \omega_{1}(t)}{2 r_{1}}(\beta T(t) V(t)+k T(t) I(t))\right\}\right\}, \\
& \varepsilon_{2}^{*}=\max \left\{\varepsilon_{2, \min }, \min \left\{\varepsilon_{2, \text { max }}, \frac{N \delta I(t) \omega_{2}(t)}{2 r_{2}}\right\}\right\} .
\end{aligned}
$$




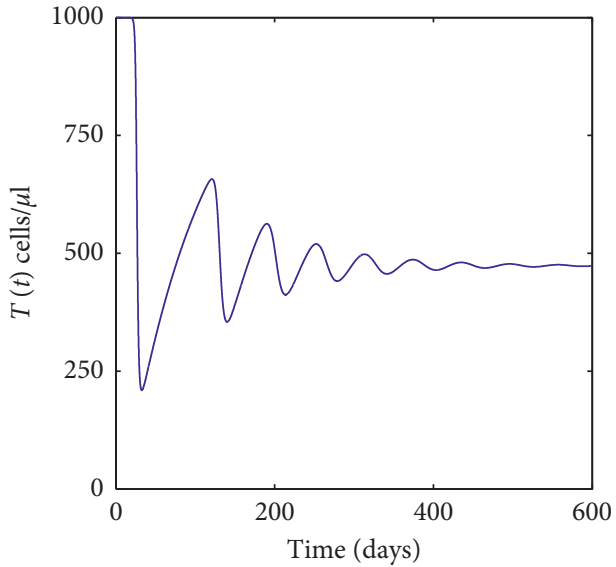

(a)

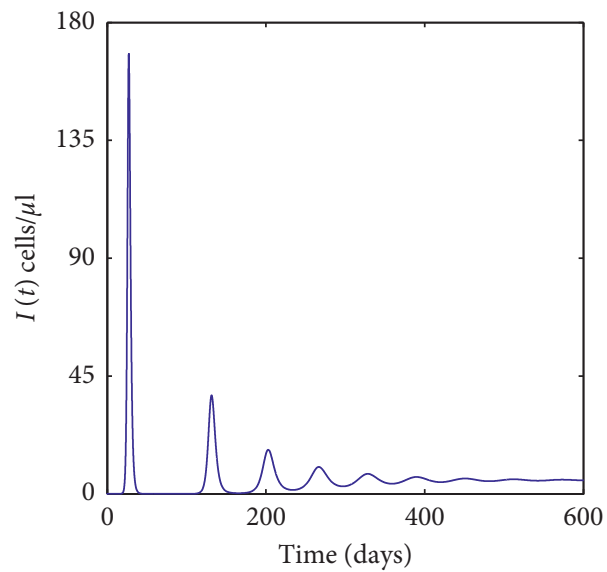

(c)

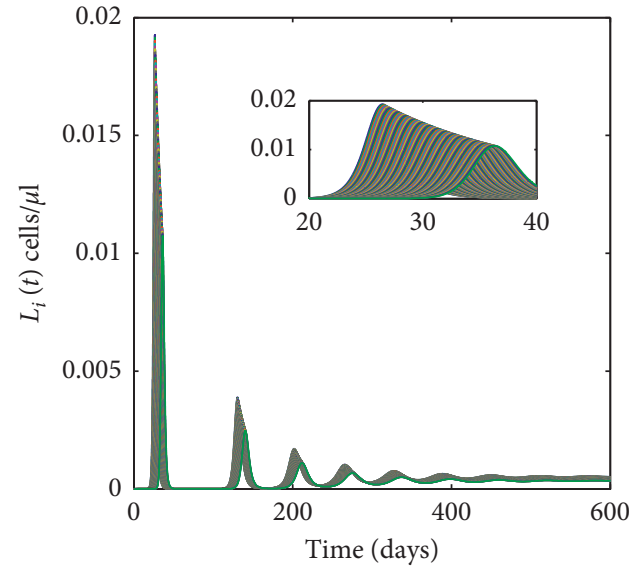

(b)

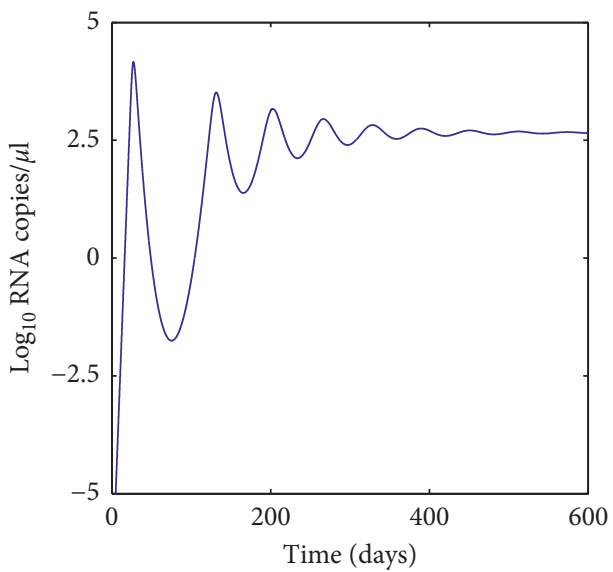

(d)

Figure 2: Predicted dynamics of uninfected CD4 ${ }^{+}$T cells, latently infected CD4 ${ }^{+}$T cells, productively infected CD4 ${ }^{+}$T cells, and viral load of the model before therapy. Using the parameter values given in the text, we have $R_{0}=2.1108>1$, which ensures that the infected equilibrium $E^{*}$ is GAS.

Proof. According to the necessary conditions of the fixed points of $\mathscr{L}$, the adjoint equations can be obtained by taking $\dot{Y}=\partial \mathscr{L} / \partial X$ with the terminal conditions $Y\left(t_{f}\right)=(0,0,0, \ldots, 0,0,0)^{\mathrm{T}}$. The optimal controls $\varepsilon_{1}^{*}$ and $\varepsilon_{2}^{*}$ can be solved from the necessary optimal conditions $\partial \mathscr{L} / \partial \varepsilon_{1}=0$ and $\partial \mathscr{L} / \partial \varepsilon_{2}=0$, respectively. That is,

$$
\begin{aligned}
\frac{\partial \mathscr{L}}{\partial \varepsilon_{1}}= & \left(\omega(t)-\frac{f}{\Delta a_{1}} \lambda_{1}(t)-(1-f) \omega_{1}(t)\right)(\beta T(t) V(t) \\
& +k T(t) I(t))+2 r_{1} \varepsilon_{1}(t)-\xi_{1}(t)+\eta_{1}(t)=0, \\
\frac{\partial \mathscr{L}}{\partial \varepsilon_{2}}= & 2 r_{2} \varepsilon_{2}(t)-N \delta I(t) \omega_{2}(t)-\xi_{2}(t)+\eta_{2}(t)=0 .
\end{aligned}
$$

Therefore, one can solve the optimal controls as follows:

$$
\begin{aligned}
\varepsilon_{1}^{*}= & \frac{1}{2 r_{1}}\left[\left(-\omega(t)+\frac{f}{\Delta a_{1}} \lambda_{1}(t)+(1-f) \omega_{1}(t)\right)(\beta T(t) V(t)\right. \\
& \left.+k T(t) I(t))+\xi_{1}(t)-\eta_{1}(t)\right], \\
\varepsilon_{2}^{*}= & \frac{1}{2 r_{2}}\left(N \delta I(t) \omega_{2}(t)+\xi_{2}(t)-\eta_{2}(t)\right) .
\end{aligned}
$$

Next, in order to derive a detailed expression for the optimal controls $\varepsilon_{1}^{*}$ and $\varepsilon_{2}^{*}$ without $\xi_{i}, \eta_{i}$, we will study all possible cases for the optimal controls.

$$
\begin{aligned}
& \text { Case I. If we consider the set } \\
& \left\{t \mid \varepsilon_{1, \text { min }}<\varepsilon_{1}^{*}(t)<\varepsilon_{1, \max }\right\}, \xi_{1}(t)=\eta_{1}(t)=0, \text { then } \\
& \text { obtain }
\end{aligned}
$$



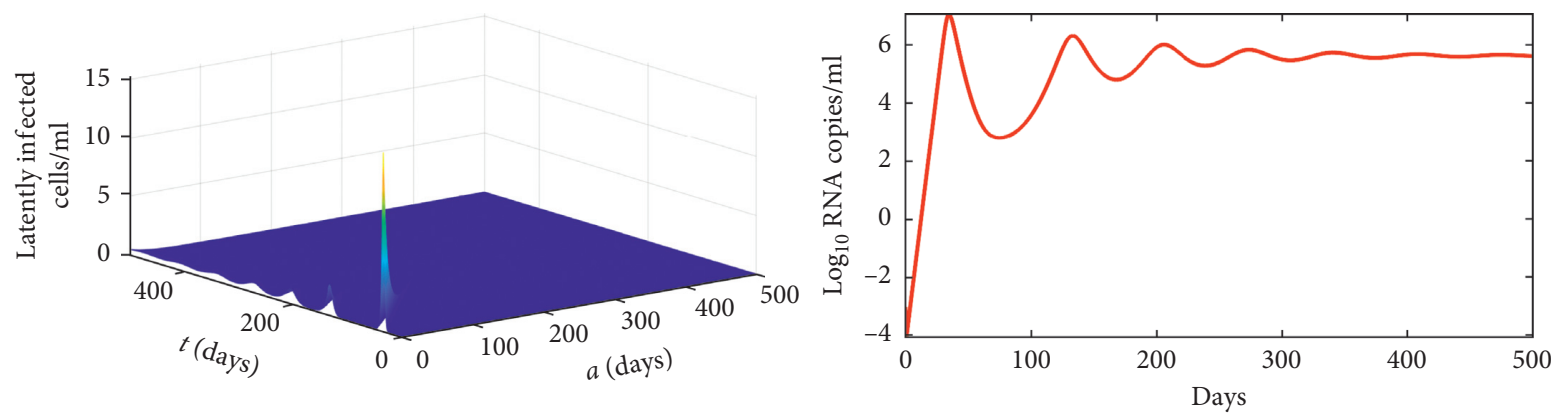

(a)
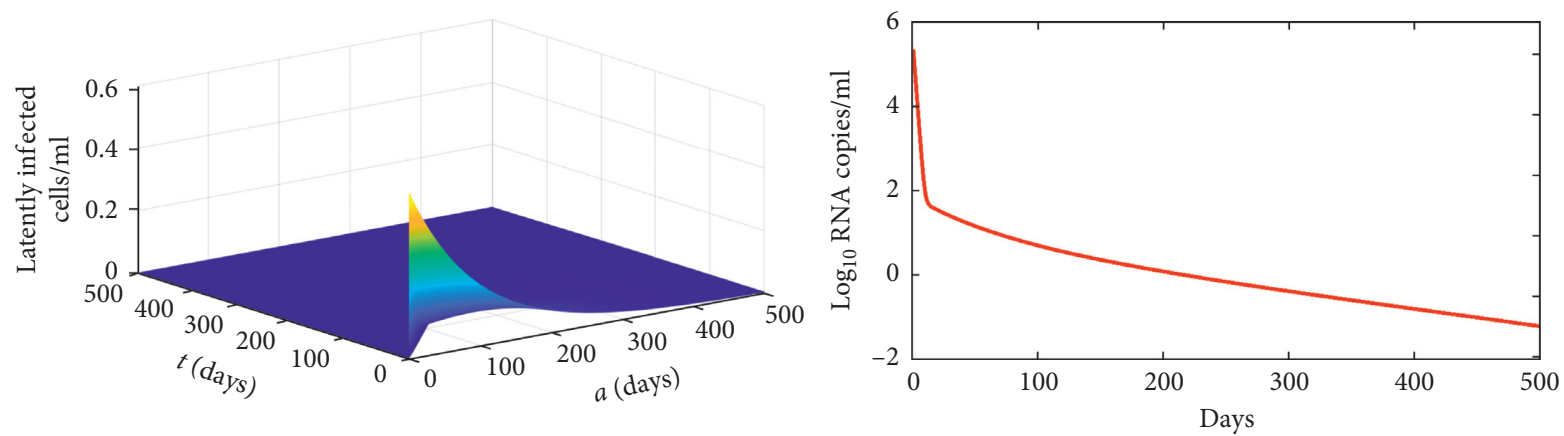

(b)
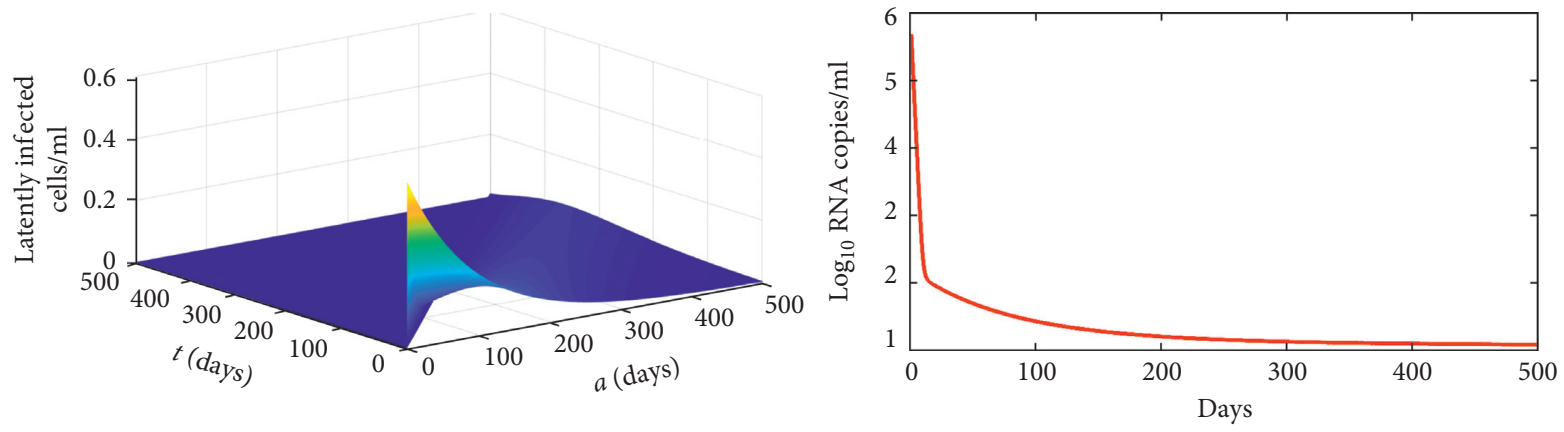

(c)

FIGURE 3: Dynamics of the virus and latent reservoir predicted by model (33). (a) The latently infected CD4 ${ }^{+}$T cells and viral load reach an infected equilibrium of model (33) without treatment and $\rho(a)-\delta_{L}(a)=0.5 a_{\min }$, where $a_{\min }=0.01 \mathrm{day}^{-1}$. (b) The latently infected CD $4^{+}$ T cells and viral level are predicted to go extinct under treatment: $\varepsilon=0.99$ and $\rho(a)-\delta_{L}(a)=0.1 a_{\min }$. (c) The latently infected CD4 ${ }^{+}$T cells and viral load persist at low levels under therapy: $\varepsilon=0.99$ and $\rho(a)-\delta_{L}(a)=a_{\min }$.

$\varepsilon_{1}^{*}=\frac{1}{2 r_{1}}\left(\frac{f}{\Delta a_{1}} \lambda_{1}+(1-f) \omega_{1}-\omega\right)(\beta T V+k T I)$.

Case II. If we consider the set $\left\{t \mid \varepsilon_{1}^{*}(t)=\varepsilon_{1, \max }\right\}, \xi_{1}(t)=0$, then we obtain

$$
\begin{aligned}
\varepsilon_{1, \max }=\varepsilon_{1}^{*} & =\frac{1}{2 r_{1}}\left[\left(\frac{f}{\Delta a_{1}} \lambda_{1}+(1-f) \omega_{1}-\omega\right)(\beta T V+k T I)-\eta_{1}\right] \\
& \leq \frac{1}{2 r_{1}}\left(\frac{f}{\Delta a_{1}} \lambda_{1}+(1-f) \omega_{1}-\omega\right)(\beta T V+k T I) .
\end{aligned}
$$

Case III. If we consider the set $\left\{t \mid \varepsilon_{1}^{*}(t)=\varepsilon_{1, \min }\right\}, \eta_{1}(t)=0$, then we obtain

$$
\varepsilon_{1, \min }=\varepsilon_{1}^{*}=\frac{1}{2 r_{1}}\left[\left(\frac{f}{\Delta a_{1}} \lambda_{1}+(1-f) \omega_{1}-\omega\right)(\beta T V+k T I)+\xi_{1}\right]
$$

$$
\geq \frac{1}{2 r_{1}}\left(\frac{f \lambda_{1}}{\Delta a_{1}}+(1-f) \omega_{1}-\omega\right)(\beta T V+k T I) .
$$

Thus, we have the optimal controls $\varepsilon_{1}^{*}$ and $\varepsilon_{2}^{*}$ in the forms of (42) by combining these three cases.

\section{Numerical Simulations}

6.1. Dynamics of the Latently Infected $C D 4^{+} T$ Cells and Viral Load. We choose parameters according to a previous study [54]: $\lambda=10^{4} \quad$ cells $\mathrm{ml}^{-1} \mathrm{day}^{-1}, \quad d_{T}=0.01 \mathrm{day}^{-1}$, $\beta=2.4 \times 10^{-8}$ virion $\mathrm{ml}^{-1} \mathrm{day}^{-1}, \quad k=2.4 \times 10^{-8}$ cell 


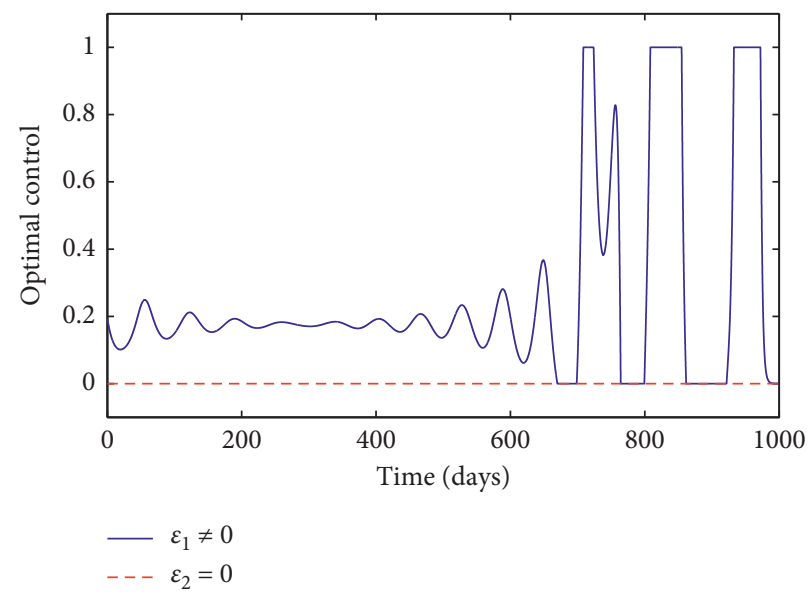

Figure 4: Optimal control strategy with $\varepsilon_{1} \neq 0$ (solid lines) and $\varepsilon_{2}=0$ (dashed lines).

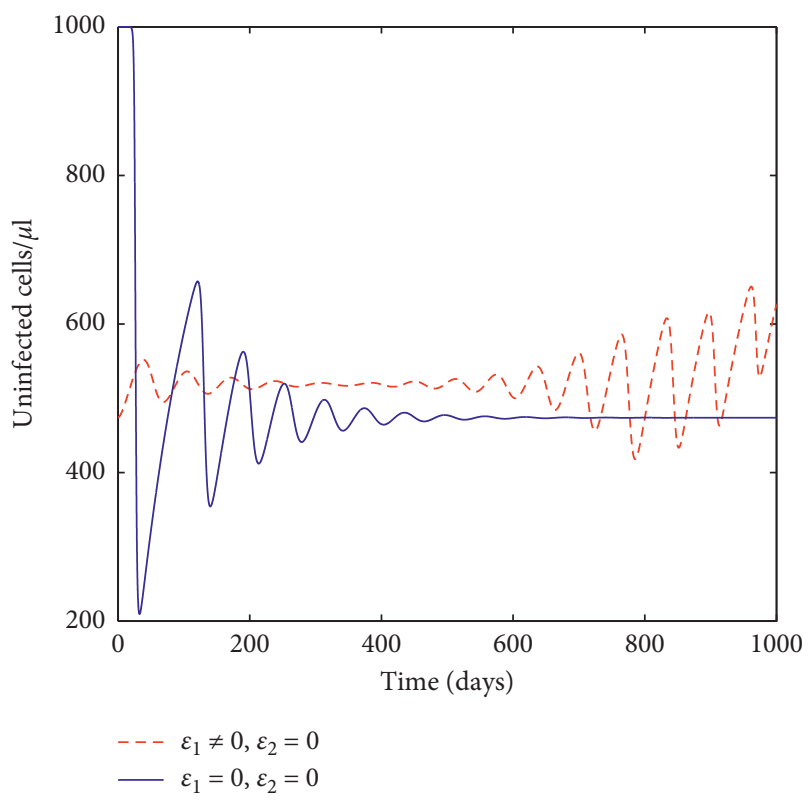

(a)

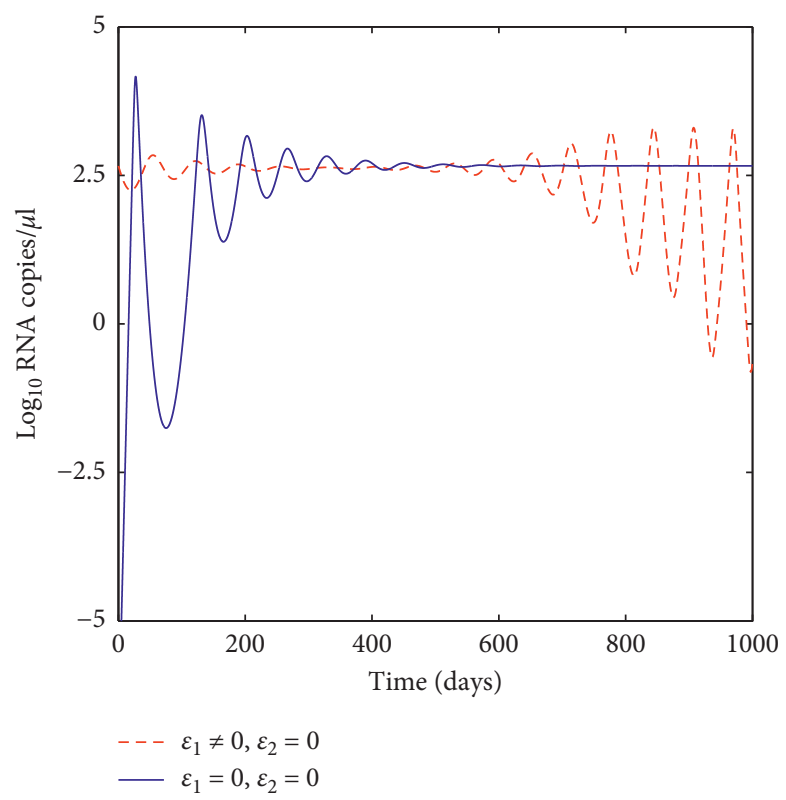

(b)

Figure 5: The level of (a) uninfected cells and (b) viral load corresponding to the optimal control strategy with $\varepsilon_{1} \neq 0$ and $\varepsilon_{2}=0$ (solid lines: without control; dashed lines: with control).

$\mathrm{ml}^{-1} \mathrm{day}^{-1}, f=0.0001, \delta=1 \mathrm{day}^{-1}, N=2000 \mathrm{cell}^{-1} \mathrm{day}^{-1}$, and $c=23 \mathrm{day}^{-1}$. Numerical simulation of model (1) with initial values $\left(T(0), L_{0}(a), I(0), V(0)\right)=\left(10^{6}, 0,0,10^{-3}\right)$ generates an infected equilibrium, which will be used as the initial value for model (1) under treatment.

We suppose that the activation rate $\alpha(a)$ of latently infected $\mathrm{CD}^{+} \mathrm{T}$ cells satisfies the exponential decay function. The function is chosen as follows:

$$
\alpha(a)=\left(a^{*}-a_{\min }\right) e^{-u a}+a_{\min },
$$

where $a_{\min }$ and $a^{*}$ denote the minimum activation rate and initial activation rate of $L(t, a)$, respectively. The parameter $u$ represents the decay parameter determining how fast $\alpha$ decreases to its minimum value. Because these parameter values remain unknown, we choose $a^{*}=0.05 \mathrm{day}^{-1}$, $u=0.01$ day $^{-1}$, and $a_{\text {min }}=0.01$ day $^{-1}$ according to ref. [44] as default values in the following simulation.

Using these parameter values, we find $R_{0}=2.1108>1$ and system (33) has the unique positive equilibrium $E^{*}=\left(T^{*}, L^{*}, I^{*}, V^{*}\right)$, where $T^{*}=4.7375 \times 10^{5}$ cells $\mathrm{ml}^{-1}$, $L^{*}=42.5322$ cells $\mathrm{ml}^{-1}, I^{*}=5.2622 \times 10^{3}$ cells $\mathrm{ml}^{-1}$, and $V^{*}=4.5758 \times 10^{5}$ RNA copies $\mathrm{ml}^{-1}$. Figures $2(\mathrm{a})-2(\mathrm{~d})$ show the dynamics of uninfected $\mathrm{CD} 4^{+} \mathrm{T}$ cells, the latent reservoir, productively infected $\mathrm{CD}^{+}{ }^{+} \mathrm{T}$ cells, and viral load of the model without treatment, respectively. To describe the stability of the latently infected $\mathrm{CD}^{+} \mathrm{T}$ cells, we choose $\gamma=\rho(a)-\delta_{L}(a)=0.5 a_{\text {min }}$. Thus, $m(a)=\alpha(a)-\gamma$. In 


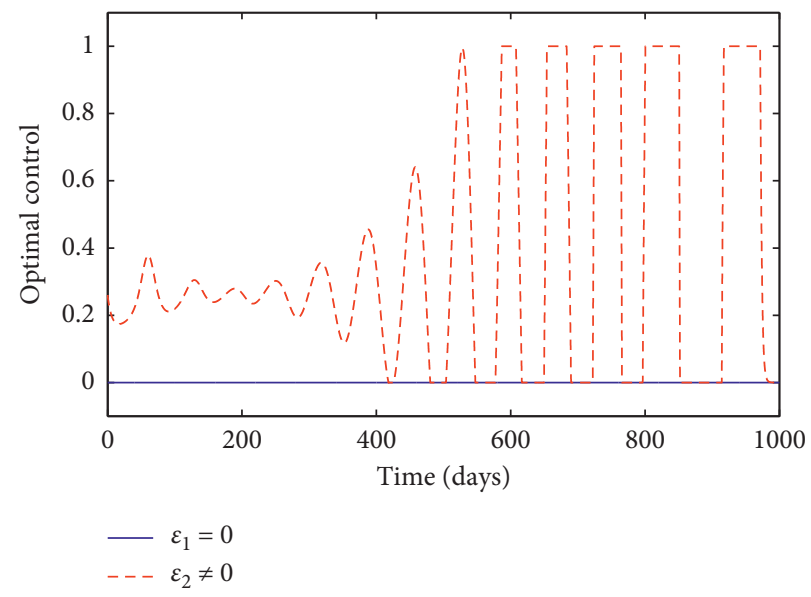

Figure 6: Optimal control strategy with $\varepsilon_{1}=0$ (solid lines) and $\varepsilon_{2} \neq 0$ (dashed lines).

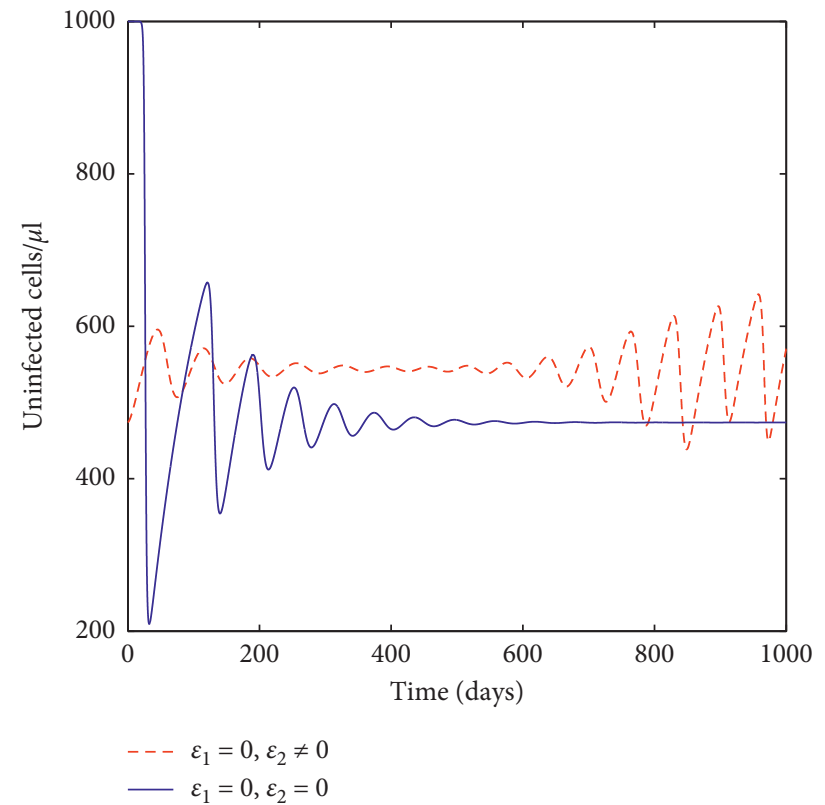

(a)

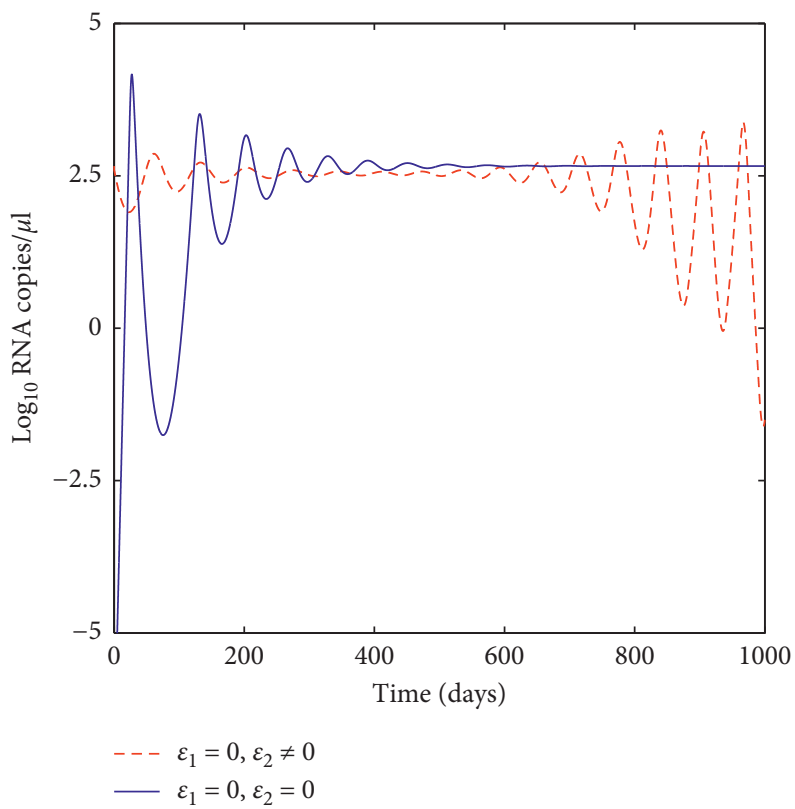

(b)

Figure 7: The level of (a) uninfected cells and (b) viral load corresponding to the optimal control strategy with $\varepsilon_{1}=0$ and $\varepsilon_{2} \neq 0$ (solid lines: without control; dashed lines: with control).

addition, we choose $n=100$ and $a_{\max }=10$ such that $\Delta a_{j}=a_{i}-a_{j-1}=0.1$, where $(j=1,2, \ldots, n)$. Thus, in the numerical simulation of the latent reservoir, age $a$ is divided into 100 equal intervals. The dynamical behaviors of latently infected $\mathrm{CD}^{+} \mathrm{T}$ cells with different ages of infection are shown in Figure 2(b).

The aim of the present section is to analyze the latently infected T cell and virus dynamics after treatment. We use an overall drug effectiveness of the combination of RTs and PIs, i.e., $\varepsilon=\left(1-\varepsilon_{1}\right)\left(1-\varepsilon_{2}\right)$ [44]. For comparison, we show the dynamics of model (1) before treatment in Figure 3(a). We find that both the virus and latently infected cells converge to positive equilibria. In Figure 3(b), we show the dynamics under therapy by using $\varepsilon=0.99$ and $\rho(a)-\delta_{L}(a)=0.1 a_{\min }$.

Both the latently infected $\mathrm{CD} 4^{+} \mathrm{T}$ cells and viral load are predicted to go extinct in this scenario. However, the decay of the latently infected $\mathrm{CD} 4^{+} \mathrm{T}$ cells and viral load is slow. In Figure 3(c), we use $\varepsilon=0.99$ but assume that $\rho(a)-\delta_{L}(a)=a_{\min }$. Both the latently infected $\mathrm{CD} 4^{+} \mathrm{T}$ cells and virus persist at a low level even under prolonged drug therapy.

6.2. Various Optimal Control Strategies. We performed numerical simulation of model (33) using the steady state of 


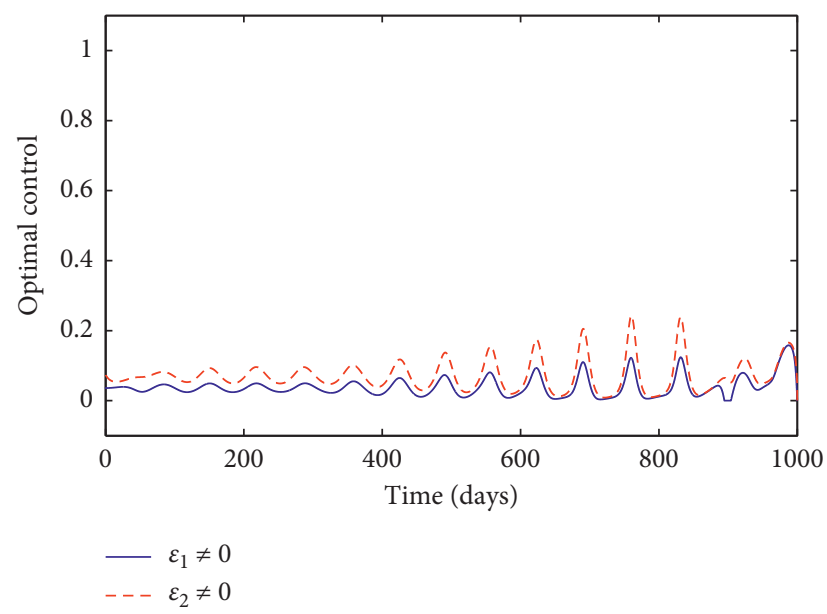

FIGURE 8: Optimal control strategy with $\varepsilon_{1} \neq 0$ (solid lines) and $\varepsilon_{2} \neq 0$ (dashed lines), where $r_{1}=r_{2}=264$.

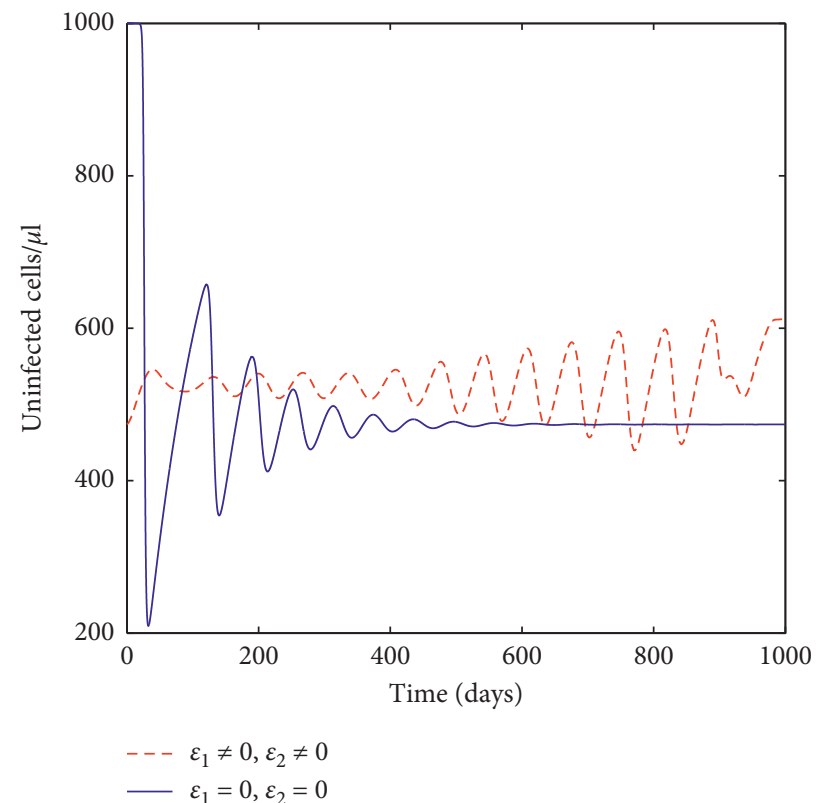

(a)

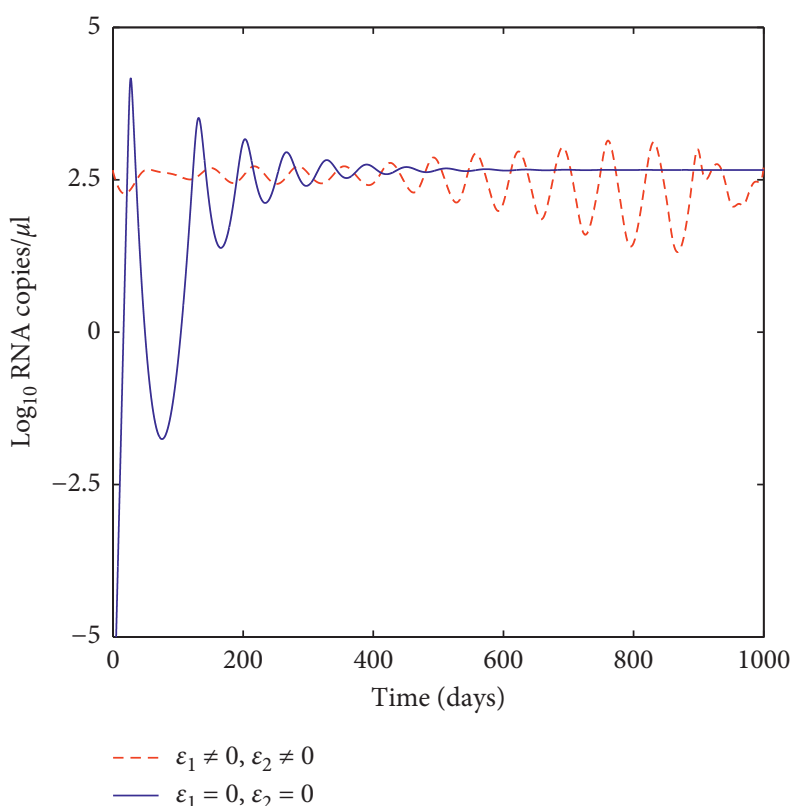

(b)

Figure 9: The level of (a) uninfected cells and (b) viral load corresponding to the optimal control strategy with $\varepsilon_{1} \neq 0$ and $\varepsilon_{2} \neq 0$. Solid lines are without control and dashed lines are with control, in which $r_{1}=r_{2}=264$.

the model before treatment as the initial condition. When the optimal control is started at the infected equilibrium, we choose parameter $q=1 / V^{*}=4.5758 \times 10^{-5}$ such that $q V^{*}=1$. The effectiveness of RTIs control $\varepsilon_{1}$ and the effectiveness of PIs $\varepsilon_{2}$ are used to optimize the objective function $J$ (given by (34)). By studying the optimal treatment strategies, different control schemes are compared. We solve the optimality system numerically by applying the forwardbackward sweep method [55] with Matlab. We choose the final time $t_{f}$ to be 1000 days. We investigate the following three cases.
Case 1: the effectiveness of RTIs $\varepsilon_{1} \neq 0$ and the effectiveness of PIs $\varepsilon_{2}=0$. In this case, we take the weight constant values $r_{1}=r_{2}=54.5$. In Figure 4 , we obtain the optimal control diagram for $\varepsilon_{1} \neq 0$ (solid lines) and $\varepsilon_{2}=0$ (dashed lines). In Figure 5, we observe that the control strategy results in an increase in the level of uninfected cells and a decrease in the viral load.

Case 2: the effectiveness of RTIs $\varepsilon_{1}=0$ and the effectiveness of PIs $\varepsilon_{2} \neq 0$. We choose the weights $r_{1}=75.8$ and $r_{2}=75.8$. In Figure 6, we show the optimal control solution for $\varepsilon_{1}=0$ (solid lines) and $\varepsilon_{2} \neq 0$ (dashed 


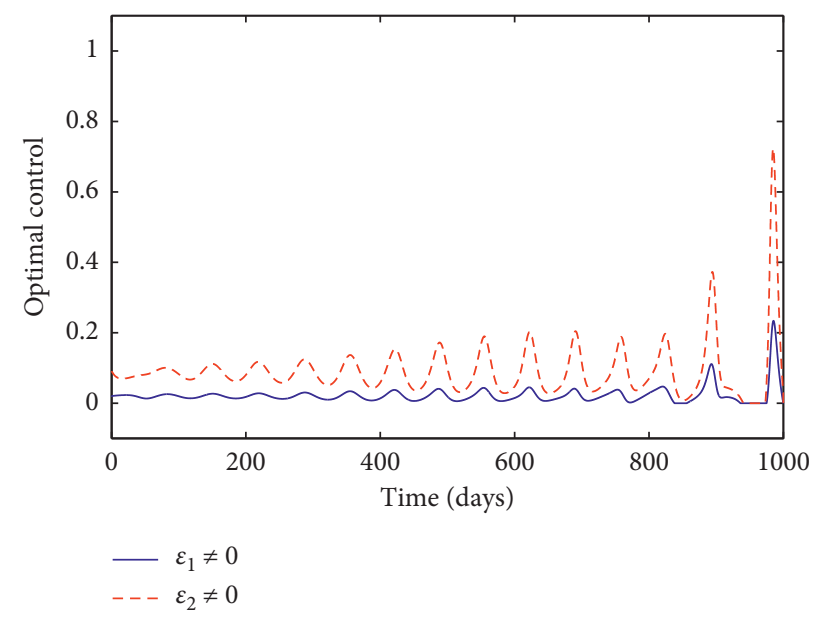

Figure 10: Optimal control strategy with $\varepsilon_{1} \neq 0$ (solid lines) and $\varepsilon_{2} \neq 0$ (dashed lines), where $r_{1}=470>r_{2}=220$.

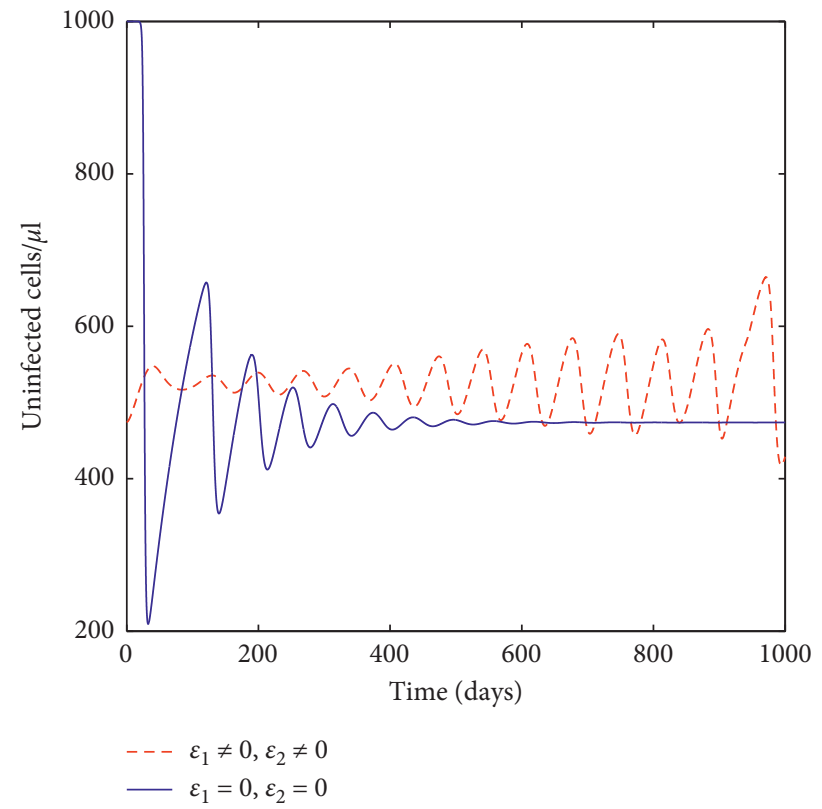

(a)

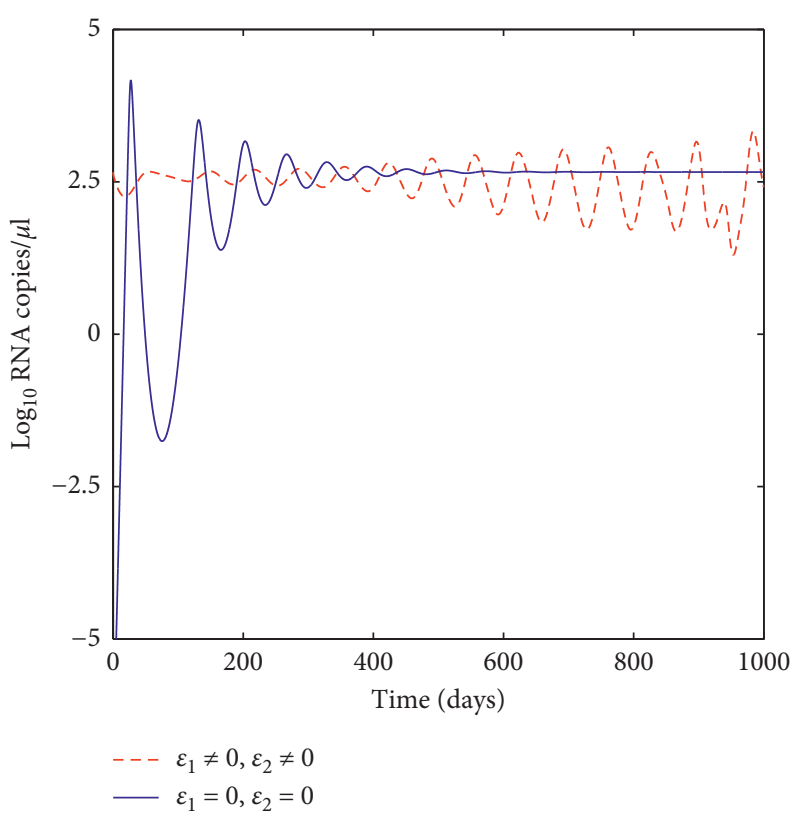

(b)

FIGURE 11: The level of (a) uninfected cells and (b) viral load corresponding to the optimal control strategy with $\varepsilon_{1} \neq 0$ and $\varepsilon_{2} \neq 0$. Solid lines are without control and dashed lines are with control, in which $r_{1}=470>r_{2}=220$.

lines). In Figure 7, we also see that the level of uninfected cells increases and viral load decreases despite oscillations.

Case 3: the effectiveness of RTIs $\varepsilon_{1} \neq 0$ and the effectiveness of PIs $\varepsilon_{2} \neq 0$.

We choose different weight constants to investigate the optimal control in this case. In Figure 8, we show the optimal control by choosing $r_{1}=r_{2}=264$ and fixing the other parameters as in Case 1. In Figure 9, we show the changes of uninfected cells and viral load under this optimal control. Uninfected cells increase and the viral load decreases under treatment. Using other combinations of the weight constants, we obtain the similar prediction. They are shown in Figures 10 and 11 for $r_{1}=470>r_{2}=220$ and in Figures 12 and 13 for $r_{1}=152<r_{2}=496$. 


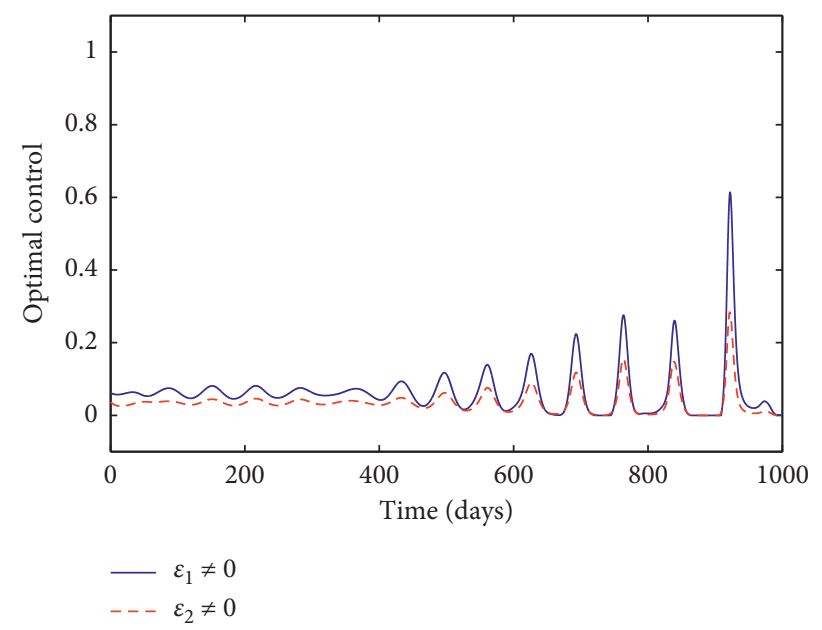

Figure 12: Optimal control strategy with $\varepsilon_{1} \neq 0$ (solid lines) and $\varepsilon_{2} \neq 0$ (dashed lines), where $r_{1}=152<r_{2}=496$.

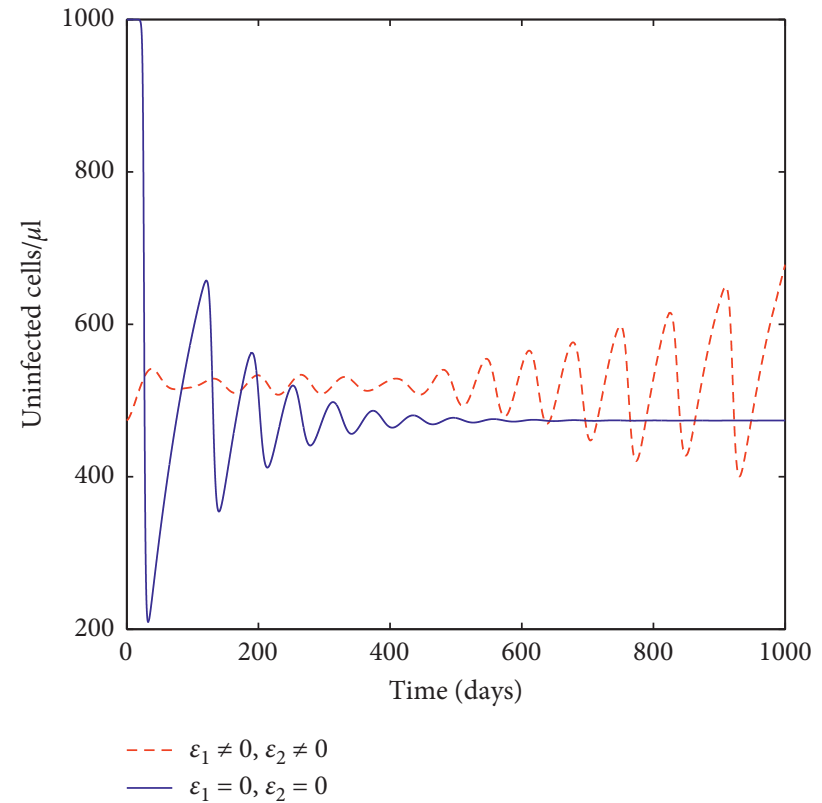

(a)

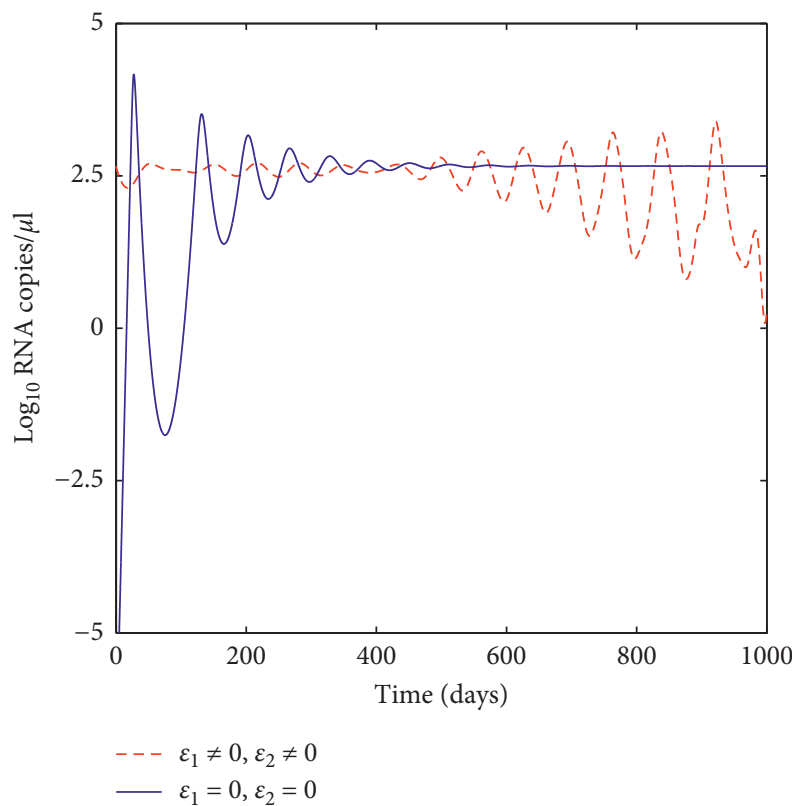

(b)

FIGURE 13: The level of (a) uninfected cells and (b) viral load corresponding to the optimal control strategy with $\varepsilon_{1} \neq 0$ and $\varepsilon_{2} \neq 0$. Solid lines are without control and dashed lines are with control, in which $r_{1}=152<r_{2}=496$.

\section{Conclusion}

HIV infection is still a serious public health problem in the world. More than 700000 people died of HIV-related causes in 2018 [56]. In addition to cell-free virus infection, HIV can also be transmitted directly from infected cells to uninfected cells. Although there is no cure for HIV infection at present, the antiretroviral drugs are effective and can control the viral level below the detection limit, largely preventing the spread of the disease [56]. HIV can remain at a low level despite long-term therapy. The latently infected $\mathrm{CD} 4^{+} \mathrm{T}$ cells are considered to be a major barrier to viral eradication. In this paper, we developed and established an age-structured model of HIV latent infection with both cell-to-cell infection and cell-to-free transmission. We showed that the solution of system (1) is positive and ultimately bounded. We derived the basic reproduction number $R_{0}$, which is an important threshold value to decide the global dynamics of the model. According to Theorems 1 and 3, the infection-free equilibrium $E^{0}$ is GAS when $R_{0}<1$, which means that HIV infection is predicted to be eliminated. To obtain the global stability of the infected equilibrium $E^{*}$, we proved the uniform persistence of system (1), followed by the global threshold dynamics using the approach of Lyapunov functionals when $R_{0}>1$. Numerical simulations were performed to illustrate the asymptotic behavior of the solution (Figure 2). We found that the balance between the activation rate and the net generation rate of latently infected $\mathrm{CD} 4^{+} \mathrm{T}$ cells plays a critical role in generating the slow decay of the latent reservoir (see Figure 3) and the persistence of low viral load during treatment. 
There are various classes of antiretroviral drugs targeting different stages of viral infection and replication. In order to achieve the optimal treatment strategies and the necessary conditions that minimize the cost of drug treatment and the viral level, we have formulated an optimal control problem using the age-structured model (33). We obtained the optimal control solutions $\varepsilon_{1}^{*}$ and $\varepsilon_{2}^{*}$ using the Pontryagin maximum principle [53]. In numerical simulations, we chose different weight constants and explored different treatment strategies (e.g., treatment with a single class of drugs or a combination of different classes of drugs). In any case, the optimal control strategy resulted in a decrease in the viral load and an increase in the level of uninfected cells.

\section{Appendix}

\section{A. Proof of Theorem 3}

In this section, we prove Theorem 3. We define a Lyapunov functional

$$
\begin{aligned}
Q(t)= & Q(T(t), L(t, \cdot), I(t), V(t))=(f \xi+1-f) \\
& \cdot\left(T(t)-T^{0}-T^{0} \ln \frac{T(t)}{T^{0}}\right)+\int_{0}^{\infty} p(a) L(t, a) \mathrm{d} a \\
& +I(t)+\frac{1-R_{02}}{N} V(t),
\end{aligned}
$$

where

$$
\begin{aligned}
p(a) & =\int_{a}^{\infty} \alpha(\theta) e^{-\int_{a}^{\theta} m(s) \mathrm{d} s} \mathrm{~d} \theta, \\
p^{\prime}(a) & =p(a) m(a)-\alpha(a), \\
p(0) & =\xi .
\end{aligned}
$$

Calculating the derivative of $Q(t)$ along the solution of system (1), we have

$$
\begin{aligned}
\frac{\mathrm{d} Q(t)}{\mathrm{d} t}= & (f \xi+1-f)\left(1-\frac{T^{0}}{T(t)}\right)\left(\lambda-d_{T} T-\beta T V-k T I\right) \\
& +\int_{0}^{\infty} p(a) \frac{\partial L(t, a)}{\partial t} \mathrm{~d} a+(1-f) \beta T V+(1-f) k T I \\
& +\int_{0}^{\infty} \alpha(a) L(t, a) \mathrm{d} a-\delta I+\frac{1-R_{02}}{N}(N \delta I-c V) .
\end{aligned}
$$

Since $\lambda=d_{T} T^{0}$ and $L(t, 0)=f \beta T V+f k T I$, we have

$$
\begin{aligned}
\frac{\mathrm{d} Q(t)}{\mathrm{d} t}= & (f \xi+1-f)\left(1-\frac{T^{0}}{T(t)}\right)\left(d_{T} T^{0}-d_{T} T(t)\right)-(f \xi+1-f) \frac{L(t, 0)}{f} \\
& +(f \xi+1-f)\left(\beta T^{0} V+k T^{0} I\right) \\
& +\int_{0}^{\infty} p(a)\left(-\frac{\partial L(t, a)}{\partial a}-m(a) L(t, a)\right) \mathrm{d} a \\
& +\frac{1-f}{f} L(t, 0)+\int_{0}^{\infty} \alpha(a) L(t, a) \mathrm{d} a-\delta I \\
& +\left(1-R_{02}\right) \delta I-\frac{1-R_{02}}{N} c V \\
= & (f \xi+1-f) d_{T} T^{0}\left(2-\frac{T^{0}}{T}-\frac{T}{T^{0}}\right)-\xi L(t, 0) \\
& +\left((f \xi+1-f) \beta T^{0}-\frac{1-R_{02}}{N} c\right) V \\
& +\left((f \xi+1-f) k T^{0}+\left(1-R_{02}\right) \delta-\delta\right) I \\
& -\left.p(a) L(t, a)\right|_{a=0} ^{a=\infty} .
\end{aligned}
$$


Note that

$$
\begin{aligned}
(f \xi+1-f) \beta T^{0}-\frac{1-R_{02}}{N} c & =\frac{c}{N}\left(\frac{(f \xi+1-f) N \beta T^{0}}{c}-1+R_{02}\right) \\
=\frac{c}{N}\left(R_{0}-1\right), & \\
(f \xi+1-f) k T^{0}+\left(1-R_{02}\right) \delta-\delta & =0, \\
p(0) L(t, 0) & =\xi L(t, 0) .
\end{aligned}
$$

Therefore, it follows from (A.4) that

$\frac{\mathrm{d} Q(t)}{\mathrm{d} t}=(f \xi+1-f) d_{T} T^{0}\left(2-\frac{T^{0}}{T(t)}-\frac{T(t)}{T^{0}}\right)+\frac{c}{N}\left(R_{0}-1\right) V(t)$.

Thus, $\mathrm{dQ}(t) / \mathrm{d} t<0$ when $R_{0}$ is less than 1 . Furthermore, $\mathrm{dQ}(t) / \mathrm{d} t=0$ if and only if $T(t)$ is exactly $T^{0}$ and $V(t)$ is 0 . It is easy to see that $\left\{E^{0}\right\}$ is the largest invariant set in $\{\mathrm{dQ}(t) / \mathrm{d} t=0\}$. Therefore, $E^{0}$ is globally attractive. This, combined with Theorem 1 , implies that $E^{0}$ is globally asymptotically stable.

\section{B. Proof of Theorem 5}

From Theorem 2, it suffices to show that $\mathscr{A}=\left\{E^{*}\right\}$. To construct a Lyapunov functional, we define $g(x)=x-1-\ln x, x>0$. Note that $g(x) \geq 0$ for all $x>0$ and $g(x)=0$ if and only if $x=1$.

Let $X(t)=(T(t), L(t, \cdot), I(t), V(t))$ be a total trajectory in $\mathscr{A}$. Note that $T(t), L(t, 0), I(t)$, and $V(t)$ are all bounded above. Moreover, by Corollary 1 , they are also bounded away from zero. Therefore, there exists $\gamma_{0}$ such that $0 \leq g(x)<\gamma_{0}$ for $x=T(t) / T^{*}, L(t, 0) / L^{*}(0), I(t) / I^{*}$, and $V(t) / V^{*}$ for all $t \in \mathbb{R}$. Since $L(t, a) / L^{*}(a)=L(t-a, 0) / L^{*}(0)$, we have $0 \leq g\left(L(t-a, 0) / L^{*}(0)\right)<\gamma_{0}$ for all $t \in \mathbb{R}$ and $a \in \mathbb{R}_{+}$.

Define a Lyapunov functional

$$
\begin{aligned}
W(t)= & W(T(t), L(t, \cdot), I(t), V(t))=W_{1}(t)+W_{2}(t) \\
& +W_{3}(t)+W_{4}(t),
\end{aligned}
$$

where

$$
\begin{aligned}
& W_{1}(t)=(f \xi+1-f) T^{*} g\left(\frac{T(t)}{T^{*}}\right), \\
& W_{2}(t)=\int_{0}^{\infty} p(a) L^{*}(a) g\left(\frac{L(t, a)}{L^{*}(a)}\right) \mathrm{d} a, \\
& W_{3}(t)=I^{*} g\left(\frac{I(t)}{I^{*}}\right), \\
& W_{4}(t)=(f \xi+1-f) \frac{\beta T^{*} V^{*}}{c} g\left(\frac{V(t)}{V^{*}}\right) .
\end{aligned}
$$

Here $p(a)$ is defined by (A.4). $W$ is well defined and is bounded.

Computing $\mathrm{d} W_{1} / \mathrm{d} t, \mathrm{~d} W_{2} / \mathrm{d} t, \mathrm{~d} W_{3} / \mathrm{d} t$, and $\mathrm{d} W_{4} / \mathrm{d} t$, together with model (1), yields

$$
\begin{aligned}
\frac{\mathrm{d} W_{1}}{\mathrm{~d} t}= & (f \xi+1-f)\left(1-\frac{T^{*}}{T(t)}\right)\left(\lambda-d_{T} T(t)-\beta T(t) V(t)\right. \\
& -k T(t) I(t)) .
\end{aligned}
$$

This, together with

$$
\begin{aligned}
\lambda & =d_{T} T^{*}+\beta T^{*} V^{*}+k T^{*} I^{*}=d_{T} T^{*}+\frac{L^{*}(0)}{f}, \\
L(t, 0) & =f \beta T(t) V(t)+f k T(t) I(t),
\end{aligned}
$$

leads to

$$
\frac{\mathrm{d} W_{1}}{\mathrm{~d} t}=(f \xi+1-f)\left(1-\frac{T^{*}}{T(t)}\right)\left(d_{T} T^{*}-d_{T} T(t)\right.
$$

$$
\begin{aligned}
& \left.+\frac{L^{*}(0)-L(t, 0)}{f}\right) \\
= & (f \xi+1-f) d_{T} T^{*}\left(2-\frac{T^{*}}{T(t)}-\frac{T(t)}{T^{*}}\right) \\
& +(f \xi+1-f) \frac{L^{*}(0)-L(t, 0)}{f}-(f \xi+1-f) \\
& +\frac{T^{*}}{T(t)} \frac{L^{*}(0)-L(t, 0)}{f} \\
= & (f \xi+1-f) d_{T} T^{*}\left(2-\frac{T^{*}}{T(t)}-\frac{T(t)}{T^{*}}\right) \\
& +\xi\left(L^{*}(0)-L(t, 0)\right)+\frac{1-f}{f}\left(L^{*}(0)-L(t, 0)\right) \\
& +(f \xi+1-f)\left(\beta T^{*} V+k T^{*} I\right) . \\
& -(f \xi+1-f)\left(\beta T^{*} V^{*}+k T^{*} I^{*}\right) \frac{T^{*}}{T(t)}
\end{aligned}
$$

Therefore, 


$$
\begin{aligned}
\frac{\mathrm{d} W_{1}}{\mathrm{~d} t}= & (f \xi+1-f) d_{T} T^{*}\left(2-\frac{T^{*}}{T(t)}-\frac{T(t)}{T^{*}}\right) \\
& +\xi\left(L^{*}(0)-L(t, 0)\right)+\frac{1-f}{f}\left(L^{*}(0)-L(t, 0)\right) \\
& -(f \xi+1-f)\left(\beta T^{*} V^{*}+k T^{*} I^{*}\right)\left(\frac{T^{*}}{T(t)}-1-\ln \frac{T^{*}}{T(t)}\right) \\
& +(f \xi+1-f) \beta T^{*} V^{*}\left(\frac{V(t)}{V^{*}}-1-\ln \frac{V(t)}{V^{*}}\right) \\
& +(f \xi+1-f) k T^{*} I^{*}\left(\frac{I(t)}{I^{*}}-1-\ln \frac{I(t)}{I^{*}}\right) \\
& +(f \xi+1-f)\left(\beta T^{*} V^{*} \ln \frac{T(t) V(t)}{T^{*} V^{*}}+k T^{*} I^{*} \ln \frac{T(t) I(t)}{T^{*} I^{*}}\right) .
\end{aligned}
$$

Secondly,

$\frac{\mathrm{d} W_{2}}{\mathrm{~d} t}=\int_{0}^{\infty} p(a)\left(1-\frac{L^{*}(a)}{L(t, a)}\right)\left(-\frac{\partial L(t, a)}{\partial a}-m(a) L(t, a)\right) \mathrm{d} a$.

Note that $p(0)=\xi$ and $p^{\prime}(a)=p(a) m(a)-\alpha(a)$. We have

$$
L^{*}(a) \frac{\partial}{\partial a}\left(g\left(\frac{L(t, a)}{L^{*}(a)}\right)\right)=\left(1-\frac{L^{*}(a)}{L(t, a)}\right)\left(\frac{\partial L(t, a)}{\partial a}+m(a) L(t, a)\right) .
$$

Using integration by parts, we have

$$
\begin{aligned}
\int_{0}^{\infty} p(a)\left(1-\frac{L^{*}(a)}{L(t, a)}\right)\left(\frac{\partial L(t, a)}{\partial a}+m(a) L(t, a)\right) \mathrm{d} a \\
=\int_{0}^{\infty} p(a) L^{*}(a) \frac{\partial}{\partial a}\left(g\left(\frac{L(t, a)}{L^{*}(a)}\right)\right) \mathrm{d} a \\
=\left.p(a) L^{*}(a) g\left(\frac{L(t, a)}{L^{*}(a)}\right)\right|_{a=0} ^{a=\infty} \\
\quad-\int_{0}^{\infty} g\left(\frac{L(t, a)}{L^{*}(a)}\right)\left(p^{\prime}(a) L^{*}(a)+p(a) L^{* \prime}(a)\right) \mathrm{d} a \\
=-\xi L^{*}(0) g\left(\frac{L(t, 0)}{L^{*}(0)}\right)+\int_{0}^{\infty} \alpha(a) L^{*}(a) g\left(\frac{L(t, a)}{L^{*}(a)}\right) \mathrm{d} a .
\end{aligned}
$$

Therefore,

$$
\begin{aligned}
\frac{\mathrm{d} W_{2}}{\mathrm{~d} t}= & \xi L^{*}(0) g\left(\frac{L(t, 0)}{L^{*}(0)}\right)-\int_{0}^{\infty} \alpha(a) L^{*}(a) g\left(\frac{L(t, a)}{L^{*}(a)}\right) \mathrm{d} a \\
= & \xi L(t, 0)-\int_{0}^{\infty} \alpha(a) L(t, a) \mathrm{d} a+\int_{0}^{\infty} \alpha(a) L^{*}(a) \ln \\
& \cdot \frac{L(t, a) L^{*}(0)}{L^{*}(a) L(t, 0)} \mathrm{d} a .
\end{aligned}
$$

Thirdly,

$$
\begin{aligned}
\frac{\mathrm{d} W_{3}}{\mathrm{~d} t}= & \left(1-\frac{I^{*}}{I(t)}\right)((1-f)(\beta T(t) V(t)+k T(t) I(t)) \\
& \left.+\int_{0}^{\infty} \alpha(a) L(t, a) \mathrm{d} a-\delta I(t)\right) .
\end{aligned}
$$

Note that

$$
\begin{aligned}
\delta I^{*} & =(1-f)\left(\beta T^{*} V^{*}+k T^{*} I^{*}\right)+\xi L^{*}(0), \\
\delta I(t) & =(1-f)\left(\beta T^{*} V^{*}+k T^{*} I^{*}\right) \frac{I(t)}{I^{*}}+\xi L^{*}(0) \frac{I(t)}{I^{*}} .
\end{aligned}
$$

It follows from (B.4) and (B.12) that

$$
\begin{aligned}
\frac{\mathrm{d} W_{3}}{\mathrm{~d} t}= & \frac{1-f}{f} L(t, 0)+\int_{0}^{\infty} \alpha(a) L(t, a) \mathrm{d} a \\
& -(1-f)\left(\beta T^{*} V^{*}+k T^{*} I^{*}\right) \frac{I(t)}{I^{*}}-\xi L^{*}(0) \frac{I(t)}{I^{*}} \\
& -(1-f) \beta T(t) V(t) \frac{I^{*}}{I(t)}-(1-f) k T(t) I^{*} \\
& -\int_{0}^{\infty} \alpha(a) L(t, a) \mathrm{d} a \frac{I^{*}}{I(t)} \\
& +(1-f)\left(\beta T^{*} V^{*}+k T^{*} I^{*}\right)+\xi L^{*}(0) .
\end{aligned}
$$

Hence,

$$
\begin{aligned}
\frac{\mathrm{d} W_{3}}{\mathrm{~d} t}= & \frac{1-f}{f} L(t, 0)+\int_{0}^{\infty} \alpha(a) L(t, a) \mathrm{d} a \\
& -(1-f)\left(\beta T^{*} V^{*}+k T^{*} I^{*}\right)\left(\frac{I(t)}{I^{*}}-1-\ln \frac{I(t)}{I^{*}}\right) \\
& -\xi L^{*}(0)\left(\frac{I(t)}{I^{*}}-1-\ln \frac{I(t)}{I^{*}}\right) \\
& -(1-f) \beta T^{*} V^{*}\left(\frac{T(t) V(t) I^{*}}{T^{*} V^{*} I(t)}-1-\ln \frac{T(t) V(t) I^{*}}{T^{*} V^{*} I(t)}\right) \\
& -(1-f) k T^{*} I^{*}\left(\frac{T(t)}{T^{*}}-1-\ln \frac{T(t)}{T^{*}}\right) \\
& -\int_{0}^{\infty} \alpha(a) L^{*}(a)\left(\frac{L(t, a) I^{*}}{L^{*}(a) I(t)}-1-\ln \frac{L(t, a) I^{*}}{L^{*}(a) I(t)}\right) \mathrm{d} a \\
& -(f \xi+1-f)\left(\beta T^{*} V^{*}+k T^{*} I^{*}\right) \\
& +(1-f) \beta T^{*} V^{*} \ln \frac{T^{*} V^{*}}{T(t) V(t)} \\
& +(1-f) k T^{*} I^{*} \ln \frac{T^{*} I^{*}}{T(t) I(t)} \alpha(a) L^{*}(a) \ln \frac{L^{*}(a)}{L(t, a)} \mathrm{d} a . \\
& (1+B .14)
\end{aligned}
$$


Lastly,

$$
\begin{aligned}
\frac{\mathrm{d} W_{4}}{\mathrm{~d} t}= & (f \xi+1-f) \frac{\beta T^{*}}{c}\left(1-\frac{V^{*}}{V(t)}\right)(N \delta I(t)-c V(t)) \\
= & (f \xi+1-f) \frac{N \delta \beta T^{*}}{c} I(t)-(f \xi+1-f) \beta T^{*} V(t) \\
& -(f \xi+1-f) \frac{N \delta \beta T^{*} I(t)}{c} \frac{V^{*}}{V(t)}+(f \xi+1-f) \beta T^{*} V^{*} .
\end{aligned}
$$

From $N \delta / c=V^{*} / I^{*}$, we obtain

$$
\begin{aligned}
\frac{\mathrm{d} W_{4}}{\mathrm{~d} t}= & (f \xi+1-f) \beta T^{*} V^{*} \frac{I(t)}{I^{*}}-(f \xi+1-f) \beta T^{*} V(t) \\
& -(f \xi+1-f) \beta T^{*} V^{*} \frac{I(t) V^{*}}{I^{*} V(t)}+(f \xi+1-f) \beta T^{*} V^{*} \\
= & (f \xi+1-f) \beta T^{*} V^{*}\left(\frac{I(t)}{I^{*}}-1-\ln \frac{I(t)}{I^{*}}\right) \\
& -(f \xi+1-f) \beta T^{*} V^{*}\left(\frac{V(t)}{V^{*}}-1-\ln \frac{V(t)}{V^{*}}\right) \\
& -(f \xi+1-f) \beta T^{*} V^{*}\left(\frac{I(t) V^{*}}{I^{*} V(t)}-1-\ln \frac{I(t) V^{*}}{I^{*} V(t)}\right) .
\end{aligned}
$$

Using equations (B.16), (B.10), (B.14), and (B.16), we have

$$
\begin{aligned}
\frac{\mathrm{d} W}{\mathrm{~d} t}= & \frac{\mathrm{d} W_{1}}{\mathrm{~d} t}+\frac{\mathrm{d} W_{2}}{\mathrm{~d} t}+\frac{\mathrm{d} W_{3}}{\mathrm{~d} t}+\frac{\mathrm{d} W_{4}}{\mathrm{~d} t} \\
= & (f \xi+1-f) d_{T} T^{*}\left(2-\frac{T^{*}}{T(t)}-\frac{T(t)}{T^{*}}\right) \\
& -(f \xi+1-f)\left(\beta T^{*} V^{*}+k T^{*} I^{*}\right) g\left(\frac{T^{*}}{T(t)}\right) \\
& +f \xi \beta T^{*} V^{*} \ln \frac{T(t) V(t)}{T^{*} V^{*}}+f \xi k T^{*} I^{*} \ln \frac{T(t) I(t)}{T^{*} I^{*}} \\
& +\int_{0}^{\infty} \alpha(a) L^{*}(a) \ln \frac{L^{*}(0)}{L(t, 0)} \mathrm{d} a \\
& -(1-f) \beta T^{*} V^{*} g\left(\frac{T(t) V(t) I^{*}}{T^{*} V^{*} I(t)}\right) \\
& -(1-f) k T^{*} I^{*} g\left(\frac{T(t)}{T^{*}}\right) \\
& -\int_{0}^{\infty} \alpha(a) L^{*}(a) g\left(\frac{L(t, a) I^{*}}{L^{*}(a) I(t)} \mathrm{d} a\right) \\
& -(f \xi+1-f) \beta T^{*} V^{*} g\left(\frac{I(t) V^{*}}{I^{*} V(t)}\right) .
\end{aligned}
$$

In view of

$$
\begin{aligned}
L(t, 0) & =f \beta T(t) V(t)+f k T(t) I(t), \\
L^{*}(0)= & f \beta T^{*} V^{*}+f k T^{*} I^{*}, \\
\int_{0}^{\infty} \alpha(a) L^{*}(a) \mathrm{d} a= & \xi L^{*}(0)=\xi f\left(\beta T^{*} V^{*}+k T^{*} I^{*}\right), \\
& f \xi \beta T^{*} V^{*}\left(\frac{T(t) V(t) L^{*}(0)}{T^{*} V^{*} L(t, 0)}-1\right)+f \xi k T^{*} I^{*}\left(\frac{T(t) I(t) L^{*}(0)}{T^{*} I^{*} L(t, 0)}-1\right)=0,
\end{aligned}
$$


we have

$$
\begin{aligned}
f \xi \beta T^{*} V^{*} \ln \frac{T(t) V(t)}{T^{*} V^{*}}+f \xi k T^{*} I^{*} \ln \frac{T(t) I(t)}{T^{*} I^{*}}+\int_{0}^{\infty} \alpha(a) L^{*}(a) \mathrm{d} a \ln \frac{L^{*}(0)}{L(t, 0)} \\
=f \xi \beta T^{*} V^{*} \ln \frac{T(t) V(t) L^{*}(0)}{T^{*} V^{*} L(t, 0)}+f \xi k T^{*} I^{*} \ln \frac{T(t) I(t) L^{*}(0)}{T^{*} I^{*} L(t, 0)} \\
=-f \xi \beta T^{*} V^{*}\left(\frac{T(t) V(t) L^{*}(0)}{T^{*} V^{*} L(t, 0)}-1-\ln \frac{T(t) V(t) L^{*}(0)}{T^{*} V^{*} L(t, 0)}\right) \\
\quad-f \xi k T^{*} I^{*}\left(\frac{T(t) I(t) L^{*}(0)}{T^{*} I^{*} L(t, 0)}-1-\ln \frac{T(t) I(t) L^{*}(0)}{T^{*} I^{*} L(t, 0)}\right) \\
\quad+f \xi \beta T^{*} V^{*}\left(\frac{T(t) V(t) L^{*}(0)}{T^{*} V^{*} L(t, 0)}-1\right)+f \xi k T^{*} I^{*}\left(\frac{T(t) I(t) L^{*}(0)}{T^{*} I^{*} L(t, 0)}-1\right) \\
=-f \xi \beta T^{*} V^{*} g\left(\frac{T(t) V(t) L^{*}(0)}{T^{*} V^{*} L(t, 0)}\right)-f \xi k T^{*} I^{*} g\left(\frac{T(t) I(t) L^{*}(0)}{T^{*} I^{*} L(t, 0)}\right) .
\end{aligned}
$$

Thus, it follows from (B.17) that

$$
\begin{aligned}
\frac{\mathrm{d} W}{\mathrm{~d} t}= & (f \xi+1-f) d_{T} T^{*}\left(2-\frac{T^{*}}{T(t)}-\frac{T(t)}{T^{*}}\right) \\
& -(f \xi+1-f)\left(\beta T^{*} V^{*}+k T^{*} I^{*}\right) g\left(\frac{T^{*}}{T(t)}\right) \\
& -f \xi \beta T^{*} V^{*} g\left(\frac{T(t) V(t) L^{*}(0)}{T^{*} V^{*} L(t, 0)}\right)-f \xi k T^{*} I^{*} g \\
& \cdot\left(\frac{T(t) I(t) L^{*}(0)}{T^{*} I^{*} L(t, 0)}\right) \\
& -(1-f) \beta T^{*} V^{*} g\left(\frac{T(t) V(t) I^{*}}{T^{*} V^{*} I(t)}\right) \\
& -(1-f) k T^{*} I^{*} g\left(\frac{T(t)}{T^{*}}\right) \\
& -\int_{0}^{\infty} \alpha(a) L^{*}(a) g\left(\frac{L^{*}(t, a) I^{*}}{L^{*}(a) I(t)}\right) \mathrm{d} a \\
& (f \xi+1-f) \beta T^{*} V^{*} g\left(\frac{I(t) V^{*}}{I^{*} V(t)}\right) .
\end{aligned}
$$

Therefore, $\mathrm{d} W(t) / \mathrm{d} t \leq 0$. Because $W$ is bounded, the alpha limit set of $x(\cdot)$ is contained in the largest invariant subset $\mathscr{M}$ in $\{\mathrm{d} W(t) / \mathrm{d} t n=q 0\}$. It is clear that $\mathscr{M}=\left\{E^{*}\right\}$. Thus, $\alpha\left(x_{0}\right)=\left\{E^{*}\right\}$, and we have $W(x(t)) \leq W\left(E^{*}\right)$. Therefore, $x(t)=E^{*}$ and $\mathscr{A}=\left\{E^{*}\right\}$.

\section{Data Availability}

This paper does not include any experimental data.

\section{Conflicts of Interest}

The authors declare that there are no conflicts of interest.

\section{Acknowledgments}

Xia Wang was supported by the NSFC (no. 11771374) and the Nanhu Scholars Program for Young Scholars of Xinyang Normal University. Libin Rong was supported by the NSF grant DMS-1758290.

\section{References}

[1] A. L. Cunningham, H. Donaghy, A. N. Harman, M. Kim, and S. G. Turville, "Manipulation of dendritic cell function by viruses," Current Opinion in Microbiology, vol. 13, no. 4, pp. 524-529, 2010.

[2] R. A. Alvarez, M. I. Barría, and B. K. Chen, "Unique features of HIV-1 spread through T cell virological synapses," PLoS Pathogens, vol. 10, no. 12, Article ID e1004513, 5 pages, 2014.

[3] J. M. Carr, H. Hocking, P. Li, and C. J. Burrell, "Rapid and efficient cell-to-cell transmission of human immunodeficiency virus infection from monocyte-derived macrophages to peripheral blood lymphocytes," Virology, vol. 265, no. 2, pp. 319-329, 1999.

[4] R. V. Culshaw, S. Ruan, and G. Webb, "A mathematical model of cell-to-cell spread of HIV-1 that includes a time delay," Journal of Mathematical Biology, vol. 46, no. 5, pp. 425-444, 2003.

[5] N. M. Dixit, A. S. Perelson, and J. M. Coffin, "HIV dynamics with multiple infections of target cells," Proceedings of the National Academy of Sciences, vol. 102, no. 23, pp. 8198-8203, 2005.

[6] P. Georgescu and Y. H. Hsieh, "Global stability for a virus dynamics model with nonlinear incidence of infection and removal," SIAM Journal on Applied Mathematics, vol. 67, no. 2, pp. 337-353, 2006. 
[7] A. S. Perelson and P. W. Nelson, "Mathematical analysis of HIV-1 dynamics in vivo," SIAM Review, vol. 41, no. 1, pp. 3-44, 1999.

[8] P. W. Nelson and A. S. Perelson, "Mathematical analysis of delay differential equation models of HIV-1 infection," Mathematical Biosciences, vol. 179, no. 1, pp. 73-94, 2002.

[9] A. S. Perelson, "Modelling viral and immune system dynamics," Nature Reviews Immunology, vol. 2, no. 1, pp. 28-36, 2002.

[10] A. S. Perelson, A. U. Neumann, M. Markowitz, J. M. Leonard, and D. D. Ho, "HIV-1 dynamics in vivo: virion clearance rate, infected cell life-span, and viral generation time," Science, vol. 271, no. 5255, pp. 1582-1586, 1996.

[11] A. S. Perelson, P. Essunger, Y. Cao et al., "Decay characteristics of HIV-1-infected compartments during combination therapy," Nature, vol. 387, no. 6629, pp. 188-191, 1997.

[12] I. A. Abela, L. Berlinger, M. Schanz et al., "Cell-cell transmission enables HIV-1 to evade inhibition by potent CD4bs directed antibodies," PLoS Pathogens, vol. 8, no. 4, Article ID e1002634, 22 pages, 2012.

[13] A. Del Portillo, J. Tripodi, V. Najfeld, D. Wodarz, D. N. Levy, and B. K. Chen, "Multiploid inheritance of HIV-1 during cellto-cell infection," Journal of Virology, vol. 85, no. 14, pp. 7169-7176, 2011.

[14] C. J. A. Duncan, R. A. Russell, and Q. J. Sattentau, "High multiplicity HIV-1 cell-to-cell transmission from macrophages to CD4+ T cells limits antiretroviral efficacy," AIDS, vol. 27, no. 14, pp. 2201-2206, 2013.

[15] M. Marsh and A. Helenius, "Virus entry: open sesame," Cell, vol. 124, no. 4, pp. 729-740, 2006.

[16] W. Mothes, N. M. Sherer, J. Jin, and P. Zhong, "Virus cell-tocell transmission," Journal of Virology, vol. 84, no. 17, pp. 8360-8368, 2010.

[17] Q. J. Sattentau, "The direct passage of animal viruses between cells," Current Opinion in Virology, vol. 1, no. 5, pp. 396-402, 2011.

[18] N. M. Sherer, M. J. Lehmann, L. F. Jimenez-Soto, C. Horensavitz, M. Pypaert, and W. Mothes, "Retroviruses can establish filopodial bridges for efficient cell-to-cell transmission," Nature Cell Biology, vol. 9, no. 3, pp. 310-315, 2007.

[19] M. Sourisseau, N. Sol-Foulon, F. Porrot, F. Blanchet, and O. Schwartz, "Inefficient human immunodeficiency virus replication in mobile lymphocytes," Journal of Virology, vol. 81, no. 2, pp. 1000-1012, 2007.

[20] A. Yakimovich, H. Gumpert, C. J. Burckhardt et al., "Cell-free transmission of human adenovirus by passive mass transfer in cell culture simulated in a computer model," Journal of Virology, vol. 86, no. 18, pp. 10123-10137, 2012.

[21] Q. Sattentau, "Avoiding the void: cell-to-cell spread of human viruses," Nature Reviews Microbiology, vol. 6, no. 11, pp. 815-826, 2008.

[22] P. Zhong, L. M. Agosto, J. B. Munro, and W. Mothes, "Cell-tocell transmission of viruses," Current Opinion in Virology, vol. 3, no. 1, pp. 44-50, 2013.

[23] L. M. Agosto, P. D. Uchil, and W. Mothes, "HIV cell-to-cell transmission: effects on pathogenesis and antiretroviral therapy," Trends in Microbiology, vol. 23, no. 5, pp. 289-295, 2015.

[24] L. M. Agosto, P. Zhong, J. Munro, and W. Mothes, "Highly active antiretroviral therapies are effective against HIV-1 cellto-cell transmission," PLoS Pathogens, vol. 10, no. 2, Article ID e1003982, 13 pages, 2014.
[25] P. Chen, W. Hübner, M. A. Spinelli, and B. K. Chen, "Predominant mode of human immunodeficiency virus transfer between $\mathrm{T}$ cells is mediated by sustained Env-dependent neutralization-resistant virological synapses," Journal of Virology, vol. 81, no. 22, pp. 12582-12595, 2007.

[26] N. Martin, S. Welsch, C. Jolly, J. A. G. Briggs, D. Vaux, and Q. J. Sattentau, "Virological synapse-mediated spread of human immunodeficiency virus type 1 between $\mathrm{T}$ cells is sensitive to entry inhibition," Journal of Virology, vol. 84, no. 7, pp. 3516-3527, 2010.

[27] D. Mazurov, A. Ilinskaya, G. Heidecker, P. Lloyd, and D. Derse, "Quantitative comparison of HTLV-1 and HIV-1 cell-to-cell infection with new replication dependent vectors," PLoS Pathogens, vol. 6, no. 2, Article ID e1000788, 12 pages, 2010.

[28] D. M. Phillips, "The role of cell-to-cell transmission in HIV infection," AIDS, vol. 8, no. 6, pp. 719-732, 1994.

[29] A. Sigal, J. T. Kim, A. B. Balazs et al., "Cell-to-cell spread of HIV permits ongoing replication despite antiretroviral therapy," Nature, vol. 477, no. 7362, pp. 95-98, 2011.

[30] P. Zhong, L. M. Agosto, A. Ilinskaya et al., "Cell-to-cell transmission can overcome multiple donor and target cell barriers imposed on cell-free HIV,” PloS One, vol. 8, no. 1, Article ID e53138, 17 pages, 2013.

[31] C. L. Althaus, A. S. De Vos, and R. J. De Boer, "Reassessing the human immunodeficiency virus type 1 life cycle through agestructured modeling: life span of infected cells, viral generation time, and basic reproductive number, R0," Journal of Virology, vol. 83, no. 15, pp. 7659-7667, 2009.

[32] C. J. Browne, "A multi-strain virus model with infected cell age structure: Application to HIV," Nonlinear Analysis: Real World Applications, vol. 22, pp. 354-372, 2015.

[33] M. A. Gilchrist, D. Coombs, and A. S. Perelson, "Optimizing within-host viral fitness: infected cell lifespan and virion production rate," Journal of Theoretical Biology, vol. 229, no. 2, pp. 281-288, 2004.

[34] G. Huang, X. Liu, and Y. Takeuchi, "Lyapunov functions and global stability for age-structured HIV infection model," SIAM Journal on Applied Mathematics, vol. 72, no. 1, pp. 25-38, 2012.

[35] X. Lai and X. Zou, "Modeling HIV-1 virus dynamics with both virus-to-cell infection and cell-to-cell transmission," SIAM Journal on Applied Mathematics, vol. 74, no. 3, pp. 898-917, 2014.

[36] X. Lai and X. Zou, "Modeling cell-to-cell spread of HIV-1 with logistic target cell growth," Journal of Mathematical Analysis and Applications, vol. 426, no. 1, pp. 563-584, 2015.

[37] P. Magal, C. C. McCluskey, and G. F. Webb, "Lyapunov functional and global asymptotic stability for an infection-age model," Applicable Analysis, vol. 89, no. 7, pp. 1109-1140, 2010.

[38] M. Shen, Y. Xiao, and L. Rong, "Global stability of an infection-age structured HIV-1 model linking within-host and between-host dynamics," Mathematical Biosciences, vol. 263, pp. 37-50, 2015.

[39] X. Wang, Y. Lou, and X. Song, "Age-structured within-host HIV dynamics with multiple target cells," Studies in Applied Mathematics, vol. 138, no. 1, pp. 43-76, 2017.

[40] X. Wang, S. Tang, X. Song, and L. Rong, "Mathematical analysis of an HIV latent infection model including both virus-to-cell infection and cell-to-cell transmission," Journal of Biological Dynamics, vol. 11, no. 2, pp. 455-483, 2017.

[41] Y. Yang, L. Zou, and S. Ruan, "Global dynamics of a delayed within-host viral infection model with both virus-to-cell and 
cell-to-cell transmissions," Mathematical Biosciences, vol. 270, pp. 183-191, 2015.

[42] L. Rong, Z. Feng, and A. S. Perelson, "Mathematical analysis of age-structured HIV-1 dynamics with combination antiretroviral therapy," SIAM Journal on Applied Mathematics, vol. 67, no. 3, pp. 731-756, 2007.

[43] V. Müller, J. F. Vigueras-Gómez, and S. Bonhoeffer, "Decelerating decay of latently infected cells during prolonged therapy for human immunodeficiency virus type 1 infection," Journal of Virology, vol. 76, no. 17, pp. 8963-8965, 2002.

[44] A. Alshorman, C. Samarasinghe, W. Lu, and L. Rong, "An HIV model with age-structured latently infected cells," Journal of Biological Dynamics, vol. 11, no. 1, pp. 192-215, 2017.

[45] H. Kim and A. S. Perelson, "Viral and latent reservoir persistence in HIV-1-infected patients on therapy," PLoS Computational Biology, vol. 2, no. 10, Article ID e135, 16 pages, 2006.

[46] M. Iannelli, "Mathematical theory of age-structured population dynamics," in Applied Mathematics Monographs 7, comitato Nazionale per le Scienze Matematiche, Consiglio Nazionale delle Ricerche (C.N.R.), Giardin, Pisa, Italy, 1995.

[47] P. Magal, "Compact attractors for time periodic age-structured population models," Electronic Journal of Differential Equations, vol. 65, pp. 1-35, 2001.

[48] J. K. Hale, Asymptotic Behavior of Dissipative Systems, American Mathematical Society, Providence, RI, USA, 1988.

[49] H. R. Thieme, "Uniform persistence and permanence for nonautonomous semiflows in population biology," Mathematical Biosciences, vol. 166, no. 2, pp. 173-201, 2000.

[50] Y. Chen, S. Zou, and J. Yang, "Global analysis of an SIR epidemic model with infection age and saturated incidence," Nonlinear Analysis: Real World Applications, vol. 30, pp. 16-31, 2016.

[51] W. Fleming and R. Rishel, Deterministic and Stochastic Optimal Controls, Springer-Verlag, Berlin, Germany, 1975.

[52] H.-D. Kwon, J. Lee, and S.-D. Yang, "Optimal control of an age-structured model of HIV infection," Applied Mathematics and Computation, vol. 219, no. 5, pp. 2766-2779, 2012.

[53] L. Pontryagin, V. G. Boltyanskii, and E. Mishchenko, The Mathematical Theory of Optimal Processes, Wiley, New York, NY, USA, 1962.

[54] L. Rong and A. S. Perelson, "Modeling HIV persistence, the latent reservoir, and viral blips," Journal of Theoretical Biology, vol. 260, no. 2, pp. 308-331, 2009.

[55] S. Lenhart and J. T. Workman, Optimal Control Applied to Biological Models, CRC Press, Boca Raton, FL, USA, 2007.

[56] WHO, HIV/AIDS, WHO, Geneva, Switzerland, 2019, https:// www.who.int/news-room/fact-sheets/detail/hiv-aids. 